\title{
Systems-level regulation of microRNA networks by miR-130/301 promotes pulmonary hypertension
}

\author{
Thomas Bertero, ${ }^{1}$ Yu Lu, ${ }^{1}$ Sofia Annis, ${ }^{1}$ Andrew Hale, ${ }^{1}$ Balkrishen Bhat, ${ }^{2}$ Rajan Saggar, ${ }^{3}$ Rajeev Saggar, ${ }^{4}$ W. Dean Wallace, ${ }^{3}$ \\ David J. Ross, ${ }^{3}$ Sara O. Vargas, ${ }^{5}$ Brian B. Graham, ${ }^{6}$ Rahul Kumar, ${ }^{6}$ Stephen M. Black, ${ }^{7}$ Sohrab Fratz, ${ }^{8}$ Jeffrey R. Fineman, ${ }^{9}$ \\ James D. West, ${ }^{10}$ Kathleen J. Haley, ${ }^{11}$ Aaron B. Waxman, ${ }^{11}$ B. Nelson Chau, ${ }^{2}$ Katherine A. Cottrill, ${ }^{1}$ and Stephen Y. Chan \\ 'Divisions of Cardiovascular Medicine and Network Medicine, Brigham and Women's Hospital, Harvard Medical School, Boston, Massachusetts, USA. Regulus Therapeutics, San Diego, California, USA. \\ ${ }^{3}$ Departments of Medicine and Pathology, David Geffen School of Medicine, UCLA, Los Angeles, California, USA. ${ }^{4}$ Department of Medicine, University of Arizona Medical Center, Tuscon, Arizona, USA. \\ ${ }^{5}$ Department of Pathology, Boston Children's Hospital, Boston, Massachusetts, USA. ${ }^{6}$ Program in Translational Lung Research, University of Colorado, Denver, Aurora, Colorado, USA. 'Vascular Biology Center, \\ Pulmonary Disease Program, Georgia Regents University, August, Georgia, USA. ${ }^{8}$ Department of Pediatric Cardiology and Congenital Heart Disease, Deutsches Herzzentrum München, Klinik an der Tech- \\ nischen Universität München, Munich, Germany. ${ }^{9}$ Department of Pediatrics, Cardiovascular Research Institute, UCSF, San Francisco, California, USA. ${ }^{10}$ Department of Medicine, Vanderbilt University Medical \\ Center, Nashville, Tennessee, USA. "Division of Pulmonary and Critical Care Medicine, Department of Medicine, Brigham and Women's Hospital, Harvard Medical School, Boston, Massachusetts, USA.
}

\begin{abstract}
Development of the vascular disease pulmonary hypertension (PH) involves disparate molecular pathways that span multiple cell types. MicroRNAs (miRNAs) may coordinately regulate PH progression, but the integrative functions of miRNAs in this process have been challenging to define with conventional approaches. Here, analysis of the molecular network architecture specific to $\mathrm{PH}$ predicted that the miR-130/301 family is a master regulator of cellular proliferation in $\mathrm{PH}$ via regulation of subordinate miRNA pathways with unexpected connections to one another. In validation of this model, diseased pulmonary vessels and plasma from mammalian models and human PH subjects exhibited upregulation of miR-130/301 expression. Evaluation of pulmonary arterial endothelial cells and smooth muscle cells revealed that miR130/301 targeted PPAR $\gamma$ with distinct consequences. In endothelial cells, miR-130/301 modulated apelin-miR-424/503FGF2 signaling, while in smooth muscle cells, miR-130/301 modulated STAT3-miR-204 signaling to promote PH-associated phenotypes. In murine models, induction of miR-130/301 promoted pathogenic PH-associated effects, while miR-130/301 inhibition prevented PH pathogenesis. Together, these results provide insight into the systems-level regulation of miRNAdisease gene networks in PH with broad implications for miRNA-based therapeutics in this disease. Furthermore, these findings provide critical validation for the evolving application of network theory to the discovery of the miRNA-based origins of $\mathrm{PH}$ and other diseases.
\end{abstract}

\section{Introduction}

Pulmonary hypertension $(\mathrm{PH})$ is an enigmatic vascular disease with poorly defined molecular origins. It is driven by various disparate triggers (hypoxia and inflammation, among others); it is marked by a pathologic imbalance of complex molecular pathways in turn promoting a number of cellular pathophenotypes (such as proliferation and vasoconstriction); and it affects multiple vascular cell types, including pulmonary arterial endothelial cells (PAECs) and smooth muscle cells (PASMCs). Identification of a regulatory factor(s) that coordinately integrates these vast molecular programs would not only offer fundamental insight into the molecular genesis of PH but also would greatly improve the strategies for therapeutic targeting of the upstream disease origins. However, the complexity of $\mathrm{PH}$ has made difficult the identification of such factors by standard reductionist strategies of experimentation.

Given their inherent pleiotropic actions to repress multiple gene targets simultaneously, microRNAs (miRNAs) may be ideal candidates to provide comprehensive and integrated control of $\mathrm{PH}$

Conflict of interest: The authors have declared that no conflict of interest exists Submitted: December 26, 2013; Accepted: May 8, 2014.

Reference information: J Clin Invest. 2014;124(8):3514-3528. doi:10.1172/JCI74773. pathogenesis. Higher-order regulation of subordinate miRNAs by upstream "master miRNAs" has begun to be described in cardiac tissue (1) and cancer (2) and may underlie the robustness of certain miRNA-based actions in health and disease. Computational approaches have been proposed to decipher systems-level regulatory motifs involving miRNAs $(3,4)$, but these theories have been challenging to study experimentally (as reviewed by ref. 5). Thus, the prospective validation of these theories in human disease in vivo has yet to be explored. In fact, to date, the miRNAs previously linked to $\mathrm{PH}$ have been associated primarily with discrete cell-specific mechanisms and phenotypes (6). For example, in diseased PASMCs, STAT3 downregulates the smooth muscle-specific miR-204 and increases cellular proliferation via activating SRC kinase (7). Alternatively, in diseased PAECs, downregulation of apelin (APLN) reduces miR$424 / 503$, thus increasing proliferation via increased FGF2 (8). Conventional molecular experimentation alone has been inadequate to discern more comprehensive, higher-order regulation of these pathways and whether these and other miRNA-based mechanisms are coordinately controlled to affect PH manifestation more robustly.

Previously, we designed a network-based bioinformatics approach (9) to predict in silico miRNAs controlling $\mathrm{PH}$ pathogenesis - via ranking of miRNAs by putative ability to recognize multiple 
targets in the same functional network of downstream PH-relevant effector genes (the "PH network," as derived by literature curation and mapped using consolidated databases of molecular interactions). In this study, we developed a more sophisticated computational method to search for overlap of functional pathways embedded in the architecture of the molecular network of PH. As a result, we aimed to identify miRNAs that are systems-level regulators of $\mathrm{PH}$, defined by an ability to integrate subordinate disease miRNAs and pathways with undefined connections to one another or to a common upstream regulator. In doing so, we identified the miR-130/301 family, the top-ranked miRNA family derived from our network-based predictions, as such a molecular integrator in $\mathrm{PH}$ - repressing its direct target PPAR $\gamma$ in order to control the STAT3-miR-204 pathway in PASMCs, the apelin-miR-424/503FGF2 pathway in PAECs, and ultimately, cellular proliferation and downstream hemodynamic manifestations of $\mathrm{PH}$ in vivo. These findings have important implications in advancing our understanding of the network hierarchy of $\mathrm{PH}$, guiding miRNA-based therapeutic strategies in this disease, and offering critical support for the application of network theory to define such higher-order regulation in complex diseases such as $\mathrm{PH}$.

\section{Results}

In silico prediction of the miR-130/301 family as a master regulator of $\mathrm{PH}$. To leverage the advancing principles of network theory for determining systems-level molecular regulators of $\mathrm{PH}$ pathogenesis, we constructed in silico a network of genes and interactions based on curated seed genes with known importance in PH (as we previously described in ref. 9) and their first-degree interactors (see Methods for details). Interactions were defined from a master list of functional molecular associations (i.e., the "consolidated interactome") catalogued from a set of databases containing all known human gene and molecular interactions (10). We refer to the complete network as the "expanded PH network," containing 249 nodes and 2,274 edges with the largest connected component encompassing all 249 nodes (Figure 1A and Supplemental Table 1; supplemental material available online with this article; doi:10.1172/JCI74773DS1).

To examine the possibility that a single miRNA may exert higher-order control over the expanded PH network as a whole, miRNAs were ranked based on the number and the spread of their predicted targets within this $\mathrm{PH}$ network. We chose the well-validated TargetScan 6.2 algorithm for miRNA target prediction, given its high degree of sensitivity and specificity relative to other algorithms of its kind (11). Because miRNAs often regulate multiple targets within the same functional pathway, especially in $\mathrm{PH}$ (9), we reasoned that a miRNA was likely to have a robust association with the $\mathrm{PH}$ network if the size of its PH-relevant target pool was larger than would be expected by chance. Thus, we ranked conserved miRNAs according to a hypergeometric analysis of their number of PH-relevant targets, accounting for expanded PH network size and the total number of targets for each miRNA. We also reasoned that a miRNA with a holistic effect on $\mathrm{PH}$ progression would have a target pool that spanned a wide segment of the network. To capture that activity, we used a spectral partitioning-based algorithm (12) to divide the expanded $\mathrm{PH}$ network into clusters based on density of edges among genes in each cluster (encircled clusters as shown in Figure 1A). MiRNAs were then ranked based on the number of distinct clusters in which they had at least 1 target. By combination of the hypergeometric analysis and the cluster-based analysis into a single score, a "miRNA spanning score" was developed to reveal the capability of a given miRNA to modulate a diverse array of processes within the expanded PH network (Supplemental Table 2). The miR-130/301 family was given the highest miRNA spanning score, both for having a large $\mathrm{PH}-$ relevant target pool (28 predicted targets depicted in Figure 1C, listed in Supplemental Table 3) and for having targets in every major network cluster ( 8 clusters). Of the 8 miR-130/301 family members listed in TargetScan, we chose 4 members to study (miR-130a/b and miR-301a/b), based on their conserved expression in rodent and human pulmonary vascular cell types. Members of this miRNA family have been studied to a modest degree, mainly as isolated factors in cancer progression $(13,14)$ and non-PH-related diseases (15-18). Notably, these miRNAs share the same seed sequence (nucleotides 2-8) and consequently putative targets (Figure 1B), but they are encoded at separate chromosomal loci, indicating that distinct transcriptional events modulate their expression. Though it was not factored into our mathematical assessment of these miRNAs, it is worth noting that the 28 predicted targets of the miR-130/301 family represented a total of 13 functional pathways embedded throughout the network, based on annotation of gene function derived from the Kyoto Encyclopedia of Genes and Genomes (KEGG), Reactome, and NCBI BioSystems databases as well as reports in the scientific literature (Figure $1 \mathrm{~A}$ and Supplemental Table 1).

Analysis of network architecture predicts important interconnections of miR-130/301 with downstream miRNA-gene target pathways in $\mathrm{PH}$. To predict important target genes, subordinate miRNAs, and related downstream pathways of miR-130/301 that may be the most influential in the progression of $\mathrm{PH}$, we performed 3 additional analyses to interrogate, as broadly as possible, the distinct mechanisms by which this miRNA family could exert its global control of PH (Figure 1D). First, to discern which of the miR130/301 targets likely carry the broadest influence of the PH network, we ranked each miR-130/301 target based on architectural features representing the span of its network influence and the robustness of its association with the miR-130/301 family (Supplemental Table 3). We refer to these features together as the "target spanning score," incorporating (a) the number of clusters in which the target had at least 1 first-degree interactor, (b) a hypergeometric P value for the overlap of the target's first-degree neighborhood and the miR-130/301 predicted target pool, and (c) the target's shortest-path betweenness centrality score, which measures the number of pairwise shortest paths between network nodes that require this target gene as a bridge. The top-ranked targets would therefore be expected to be highly influential in the context of $\mathrm{PH}$ (a high betweenness centrality score and a large number of interacting clusters) and highly sensitive to the regulatory effects of the miR-130/301 family (carrying a first-degree neighborhood of genes targeted multiple times by this miRNA family). The TGFBMP pathway members SMAD4 and SMAD5 as well as PPAR $\gamma$ were included among the top 5 genes. Importantly, their rankings together were consistent with prior reports linking upstream BMP signaling to PPAR $\gamma$ and PASMC proliferation (19) and other 
A

\begin{tabular}{|c|c|}
\hline Angiopoietin Signaling & Prostacyclin \\
\hline Apelin Signaling & Protein Processing in the ER \\
\hline Apoptosis & Regulation of Actin Cytoskeleton \\
\hline Calcium Signaling Pathway & Rho Kinase \\
\hline Cation Channel Activity & Serotonin Signaling \\
\hline EGF Pathway & TGF- $\beta$ Signaling \\
\hline Endothelin Pathway & TNF Signaling Pathway \\
\hline Fibrosis & Thromboxane A2 Receptor Signal \\
\hline FoxO Family Signaling & VEGF Signaling \\
\hline Hypoxia and Oxygen Signaling & Vascular Inflammation \\
\hline Mitochondrial Metabolism & Vascular Smooth Muscle Contracti \\
\hline Nitric Oxide Synthesis & p53 Signaling Pathway \\
\hline PDGF Pathway & Other \\
\hline
\end{tabular}

B

miR-130a cagugcaAUGUUAAAAgGgcaU miR-130b CAGUGCAAUGAUGAAAGGGCAU miR-301a CAGUGCAAUAGUAUUGUCAAAGC miR-301b CAGUGCAAUGAUAUUGUCAAAGC

C

Angiopoietin signaling
Cation channel activity
Endothelin pathway
PDGF pathway
PPAR signaling pathway
Protein processing in the ER
Hypoxia and oxygen signaling
Mitochondrial metabolism
Nitric oxide synthesis
TGF- $\beta$ Signaling
Vascular inflammation
Vascular smooth muscle
contraction
Rho kinase
Other

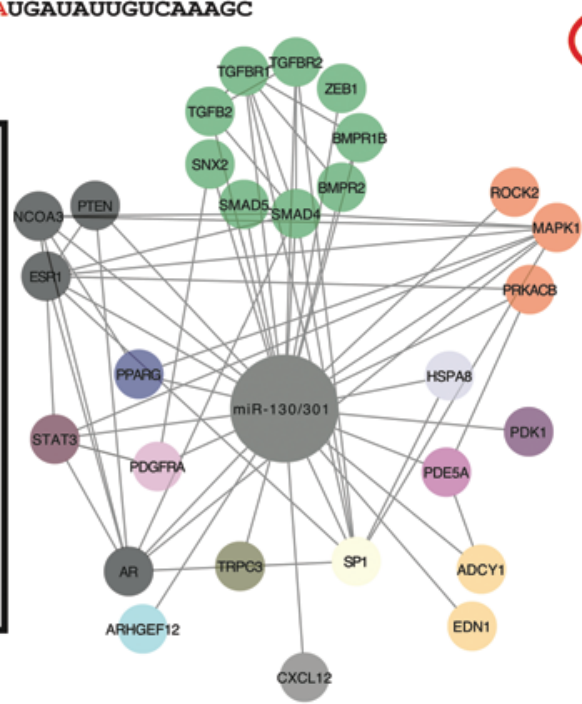

D

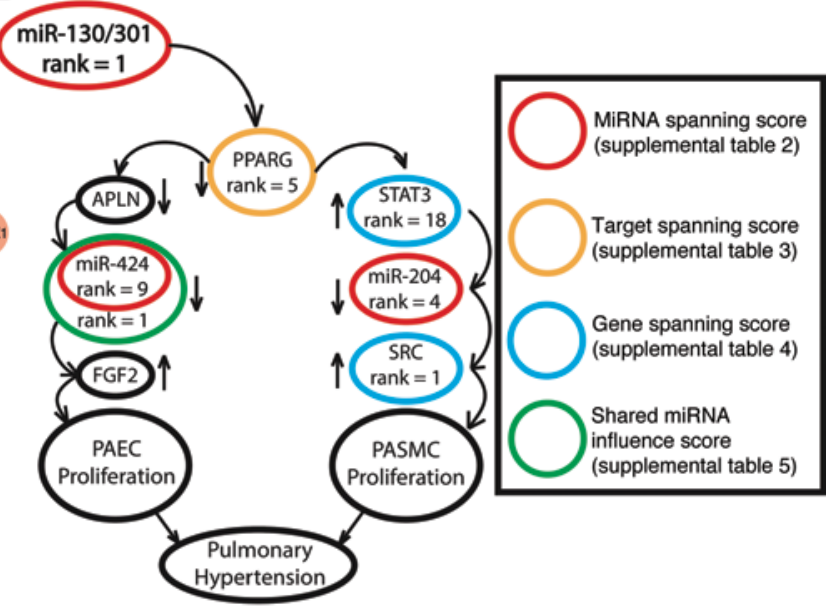

Figure 1. In silico network-based analysis identifies the miR-130/301 family as a master regulator of subordinate miRNA pathways and PH. (A) The expanded $\mathrm{PH}$ network is color-coded according to functional pathway. Encircled areas represent architectural clusters based on a spectral partition-based clustering algorithm. The miR-130/301 family was ranked the highest by a miRNA spanning score, reflecting the most robust systems-level control over the expanded PH network as a whole. Direct targets of the miR-130/301 family ( 28 enlarged nodes) span all 8 gene clusters and 13 functional pathways. (B) The miR-130/301 family members share the same seed sequence (in red). (C) Direct targets of the miR-130/301 family, color-coded by functional pathway. (D) Predictions of downstream pathways connected to the miR-130/301 family and carrying the broadest influence on the expanded PH network, according to 4 scoring algorithms: (a) MiRNA spanning score: MiRNAs were ranked based on their network influence (see also Supplemental Table 2). (b) Target spanning score: Targets of miR-130/301 were ranked based on their network influence and the robustness of their relationship to the miR-130/301 family (see also Supplemental Table 3). (c) Gene spanning score: All network nodes were ranked based on their network influence (see also Supplemental Table 4). (d) Shared miRNA influence score: MiRNAs were ranked based on the overlap of their PH-relevant target pool with the miR-130/301 family (see also Supplemental Table 5).

reports linking PPAR $\gamma$ directly to PH development (20-22). However, the full spectrum of the regulation and downstream actions of PPAR $\gamma$ in PH remains undescribed (23). Thus, we chose to focus further on the downstream biology of PPAR $\gamma$ in relation to the miR-130/301 family, which seemed most relevant for an in-depth network-based interrogation.
Second, we reasoned that, regardless of its status as a direct target of the miR-130/301 family, a highly influential gene in the expanded $\mathrm{PH}$ network with a wide sphere of network influence should share functional overlap with the miR-130/301 family, which regulates the network on a similarly broad scale. Thus, to cast an even wider net for capturing all genes highly active in 

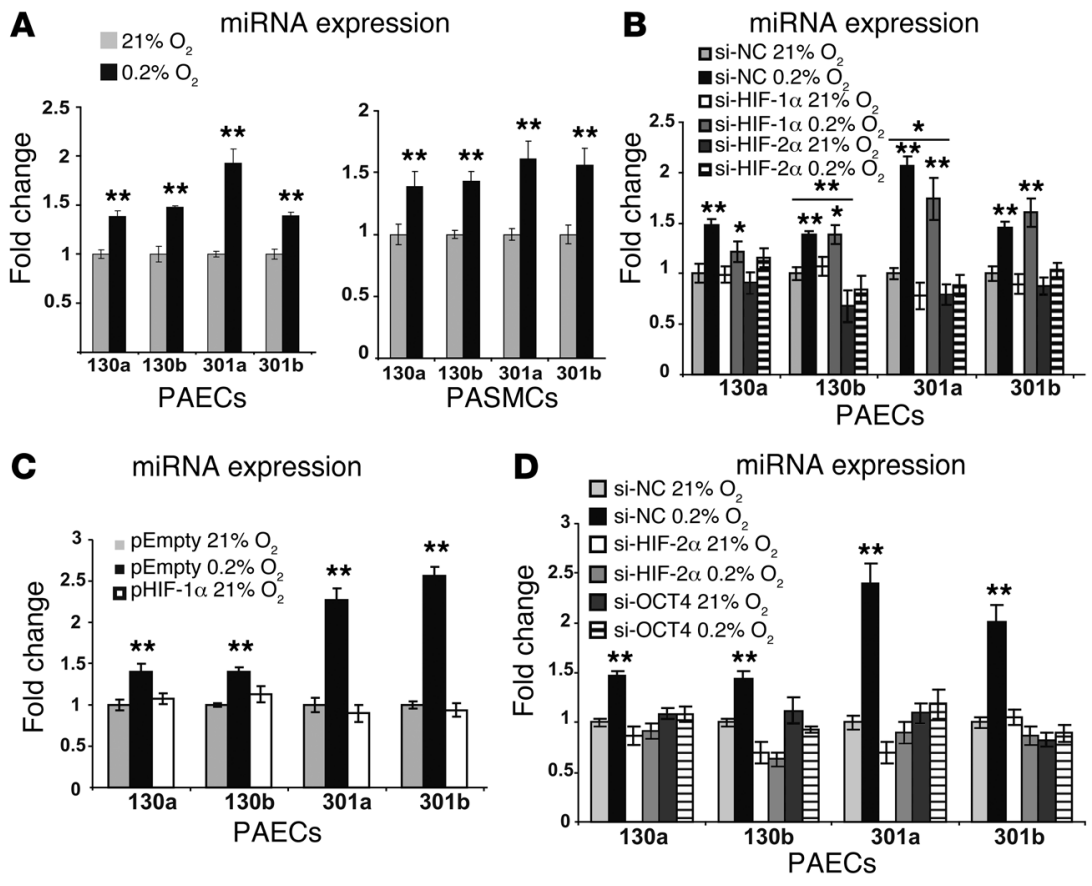

Figure 2. Upregulation of the miR-130/301 family by hypoxia is mediated by HIF-2 $\alpha$ and POU5F1/OCT4. (A) As demonstrated by RT-qPCR, hypoxia ( $0.2 \% \mathrm{O}_{2}$ for 24 hours) upregulated the miR-130/301 family in both human PAECs and PASMCs as compared with normoxia (21\% $\mathrm{O}_{2}$ for 24 hours). (B) In PAECs transfected with siRNA control (si-NC) or siRNA specific for HIF-1 $\alpha$ (si-HIF-1 $\alpha$ ), hypoxia upregulated all miR-130/301 family members compared with normoxia. In contrast, in normoxia, miR-130/301 family members were downregulated during HIF-2 $\alpha$ knockdown (si-HIF-2 $\alpha$ ) compared with control. Moreover, during HIF-2 $\alpha$ knockdown, family members were not induced by hypoxia. (C) In normoxic PAECs, lentiviral transduction with a constitutively expressed HIF-1 $\alpha$ transgene carrying a proline-to-alanine mutation (pHIF1) did not alter miRNA expression compared with transduction with empty vector (pEmpty) alone. In contrast, hypoxia upregulated all miR-130/301 family members compared with normoxia in the presence of pEmpty alone. (D) During HIF-2 $\alpha$ (si-HIF-2 $\alpha$ ) or POU5F1/ OCT4 (si-OCT4) knockdown in PAECs, miR-130/301 family members were not induced by hypoxia, in contrast to cells transfected with siRNA control (si-NC). In each panel, for each miRNA, mean expression in normoxic control groups $\left(21 \% \mathrm{O}_{2}\right.$ in $\mathrm{A}$; si-NC $21 \% \mathrm{O}_{2}$ in $\mathbf{B}$ and $\mathbf{D}$; pEmpty $21 \% \mathrm{O}_{2}$ in $\mathbf{C}$ ) was assigned a fold change of 1 , to which relevant samples were compared. Data are presented as mean $\pm \mathrm{SD}\left({ }^{*} P<0.05 ;{ }^{* *} P<0.01\right)$.

the expanded PH network as a whole, we ranked all nodes in the expanded PH network according to their influence ("gene spanning score"; Supplemental Table 4). Again, genes were scored on 3 architectural features reflecting their influence on the network as a whole: (a) their shortest-path betweenness centrality score, (b) the number of architectural clusters in which they had at least 1 first-degree interactor, and (c) the degree of interactions by each gene in the network. The top-ranked gene was $S R C$, a nonreceptor tyrosine kinase known to be associated with both STAT3 (the 18th ranked gene in this list) and miR-204 (the 4th ranked miRNA by miRNA spanning score; Supplemental Table 2) in order to control PASMC proliferation in PH (7). Thus, while none of these factors has been linked to the miR-130/301 family, the substantial overlap of functional influence in the expanded $\mathrm{PH}$ network between STAT3/miR-204/SRC and the miR-130/301 family predicted their functional association.

Finally, to capture additional miRNAs that may be associated with the miR-130/301-dependent regulation of $\mathrm{PH}$, we ranked all conserved miRNAs represented in the TargetScan algorithm ac- cording to a hypergeometric analysis of the overlap of their PH-relevant target pools with the $\mathrm{PH}$ relevant target pool of the miR-130/301 family ("shared miRNA influence score"; Supplemental Table 5). The top-ranked set of miRNAs included miR-424, a miRNA also ranked highly (top 10) by miRNA spanning score (Supplemental Table 2). Thus, although miR-424 had not previously been associated with the miR-130/301 family, these rankings indirectly connected these miRNAs by their broad spheres of influence in PH. Specifically, along with miR-503, miR-424 is known to be dysregulated in $\mathrm{PH}$ by alterations in the peptide ligand APLN, thus inciting upregulation of FGF2 in PAECs (8). Furthermore, while itself never previously connected to the miR-130/301 family, APLN has been associated with both miR-424 and miR-503 (8) as well as PPAR $\gamma$ in PAECs (24). Consequently, the generation of the expanded PH network coupled with computational analyses of network architecture not only predicted the broad influence of the miR-130/301 family in $\mathrm{PH}$ pathogenesis but also provided a detailed map of integrated miRNA-gene target pathways by which this miRNA family may exert its influence.

The miR-130/301 family is upregulated by multiple triggers of $\mathrm{PH}$, including hypoxia via a dependence on HIF-2 $\alpha$ and POU5F1/OCT4. To begin to validate this molecular model, we wanted to determine whether the miR-130/301 family is coordinately regulated by known triggers of $\mathrm{PH}$. First, we investigated whether genes previously linked to hereditary pulmonary arterial hypertension (PAH) (as reviewed by ref. 25) may regulate this miRNA family. In both human PAECs and PASMCs (Supplemental Figure 1, C and D), we found that siRNA-mediated knockdown of $B M P R 2$ or CAV1 increased miR-130/301 expression, while inhibition of ALK1, ENG, KCNK3, and SMAD9 had negligible effects on this miRNA family. Next, we found that multiple acquired PH triggers, including hypoxia (Figure 2A) and the inflammatory cytokines IL-1 1 (Supplemental Figure 1A) and IL-6 (Supplemental Figure 1B), upregulate miR-130/301 expression as a whole but with some specific differences in family member profile in each context. Consistent with our results (Supplemental Figure 1 and Supplemental Table 2), some miR-130/301 family members have been reported to be transcribed by an NF- $\mathrm{kB}$-dependent mechanism during inflammatory cytokine stimulation (13). Alternatively, we found that knockdown of a master transcription factor of hypoxia, HIF- $2 \alpha$, but not HIF-1 $\alpha$, prevented the increase of these miRNAs in hypoxia (Figure 2B). In corroboration, forced expression of HIF-1 $\alpha$ was insufficient to upregulate any miR-130/301 family members (Figure 2C). No identifiable binding sites were predicted for HIF-2 $\alpha$ in the promoter region of any miR-130/301 family members (Supplemental Table 6). However, a putative binding sequence was predicted in all family member promoter sites for the transcription factor POU5F1/OCT4, a factor known 
A miRNA expression

C

miRNA expression

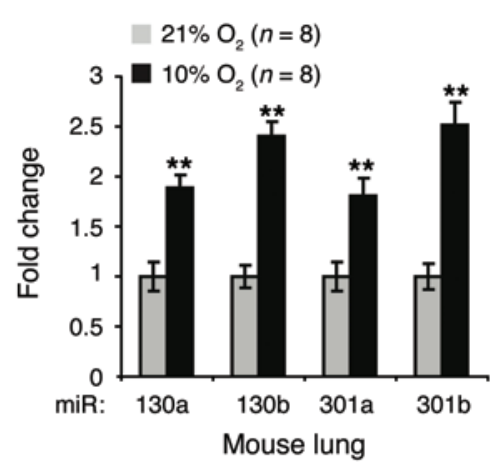

D

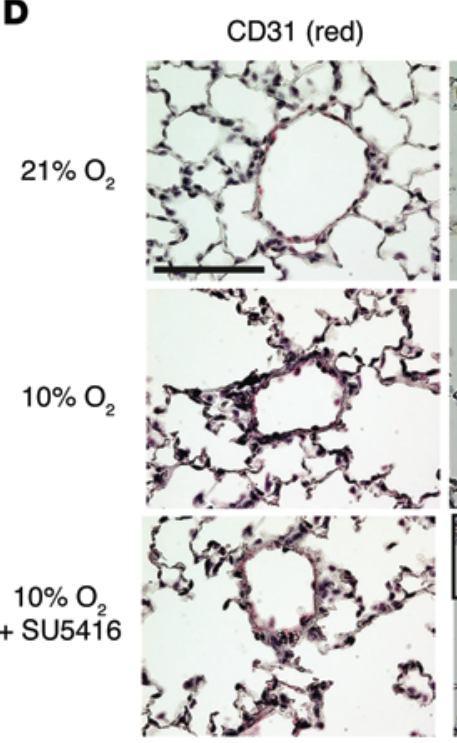

G

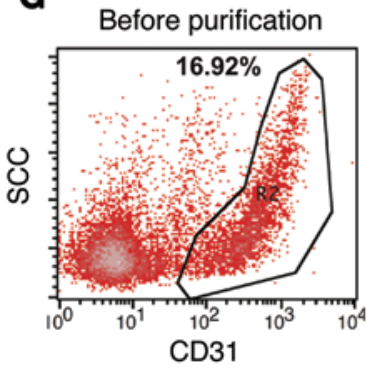

B

miRNA expression

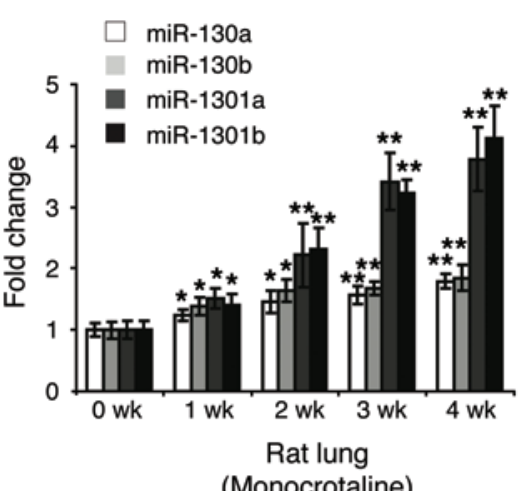

(Monocrotaline)

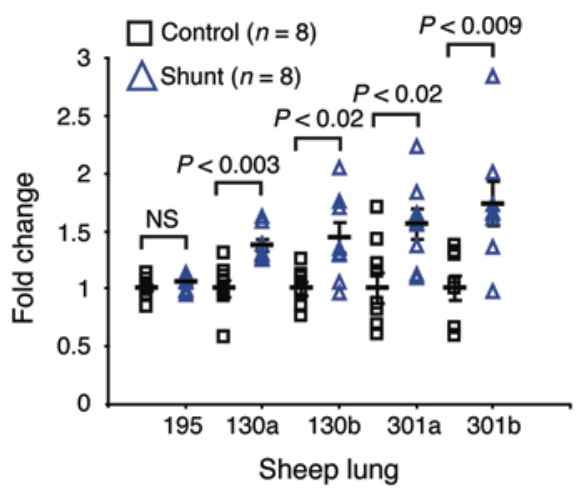

E Vascular miR-130a expression
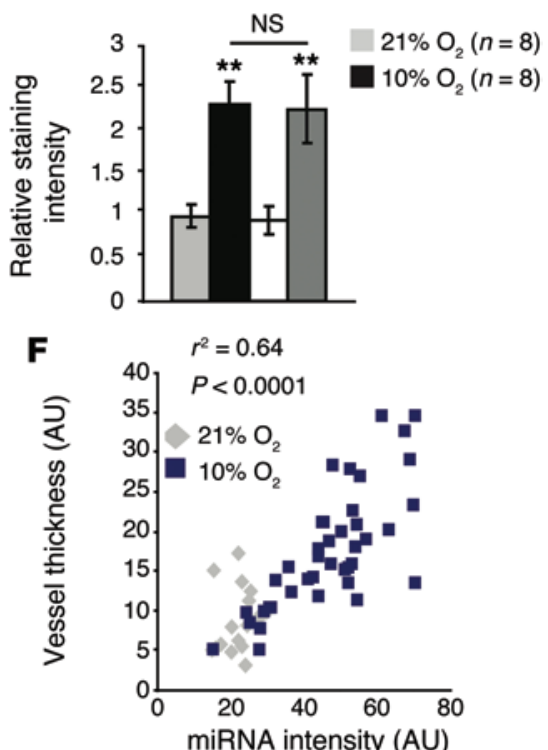

miR-130a probe (blue) $\quad \alpha$-SMA (red)

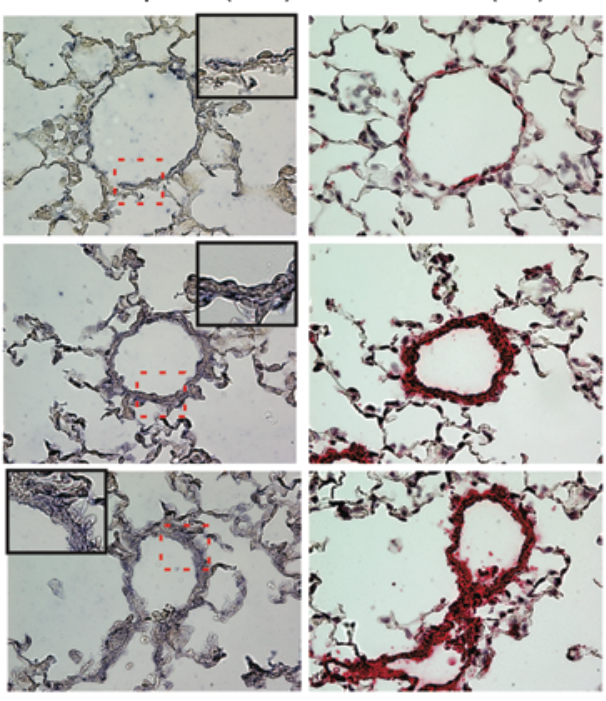

H
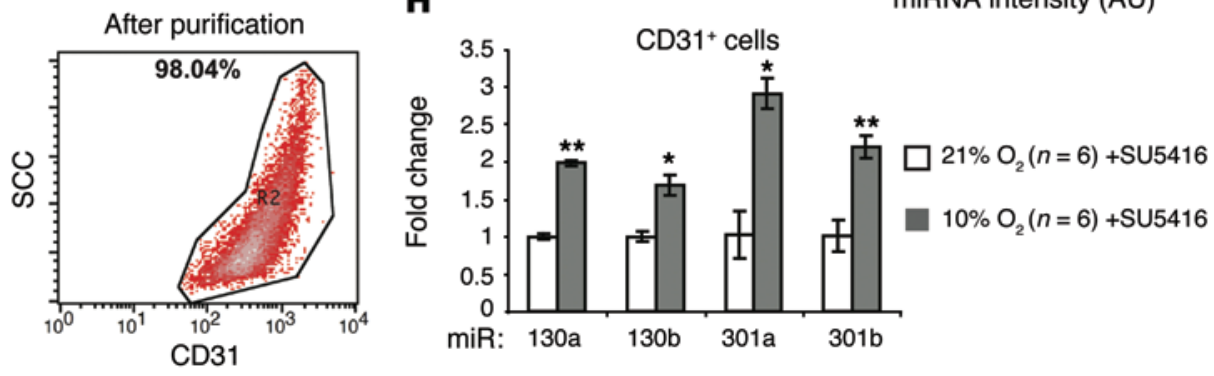

Figure 3. The miR-130/301 family is induced in multiple models of PH. (A) By RT-qPCR, miR-130/301 was increased in lungs of mice suffering from PH induced by SU5416 administration with chronic hypoxia $\left(10 \% \mathrm{O}_{2}\right.$ for 3 weeks; $\left.n=12\right)$ versus normoxia $\left(21 \% \mathrm{O}_{2} ; n=12\right)$. (B) MiR-130/301 progressively increased in lungs of rats with monocrotaline-induced $\mathrm{PH}$ ( $n=8$ rats/treatment group). (C) MiR-130/301 was increased in lungs of juvenile sheep with shunt-induced PAH (Shunt; $n=8$ ) as compared with sham controls $(n=8)$. For each experiment, mean expression in controls was assigned a fold change of 1 , to which relevant samples were compared. In situ hybridization of serially sectioned mouse lung (D) and quantification of pulmonary vessels less than $100 \mu \mathrm{m}$ in diameter ( 5 vessels per mouse) (E) revealed that miR-130a was upregulated in small pulmonary vessels $(<100 \mu \mathrm{m})$ exposed to hypoxia with or without SU5416 $\left(10 \% \mathrm{O}_{2}\right)$ as compared with normoxia $\left(21 \% \mathrm{O}_{2}\right)$. (F) Intensity of miR-130a stain in situ positively correlated with pulmonary vessel thickness. (G) Representative flow cytometric analysis of CD31 expression in mouse lung endothelial cells before (left panel) and after CD31-specific MACS purification (right panel). (H) By RT-qPCR, miR-130/301 was increased in CD31-positive pulmonary vascular endothelial cells of mice exposed to SU5416 with hypoxia $\left(10 \% \mathrm{O}_{2} ; n=6\right)$ versus normoxia $\left(21 \% \mathrm{O}_{2} ; n=6\right)$. Significant differences are indicated ( $\left.{ }^{*} P<0.05 ;{ }^{* *} P<0.01\right)$. In physiologic experiments $(\mathbf{A}-\mathbf{C}$ and $\mathbf{H})$, data are expressed as mean \pm SEM, while in the in situ quantitations (E and $\mathbf{F}$ ), data are expressed as mean \pm SD. Tissue scale bar: $50 \mu \mathrm{m}$.

to be modulated by HIF- $2 \alpha$ but not HIF-1 $\alpha$ (26) and known to be upregulated in PAH (27). Consequently, we found that POU5F1/ OCT4 was upregulated in hypoxia and was dependent on HIF-2 $\alpha$ in PAECs and PASMCs (Supplemental Figure 2, A-D). Furthermore,
siRNA knockdown of POU5F1/OCT4 inhibited miR-130/301 induction by hypoxia in PAECs (Figure 2D) and PASMCs (Supplemental Figure 2E), thus establishing the critical importance of the HIF-2 $\alpha$-POU5F1/OCT4 regulatory axis in miR-130/301 expres- 
A
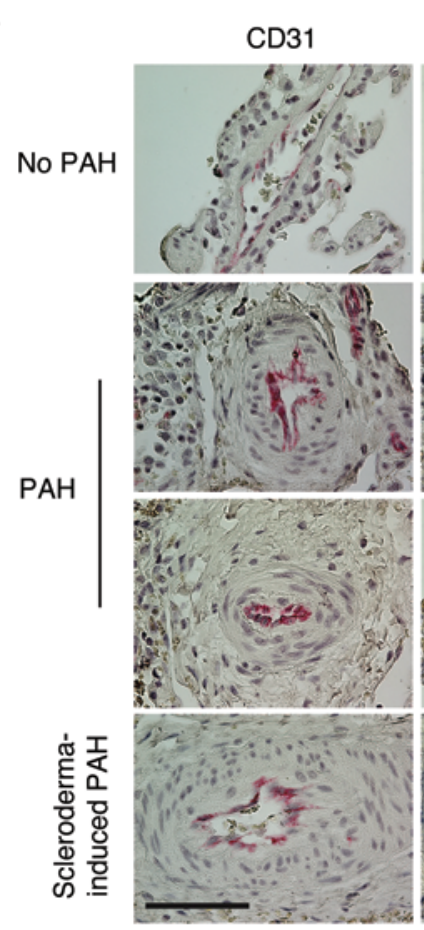

C

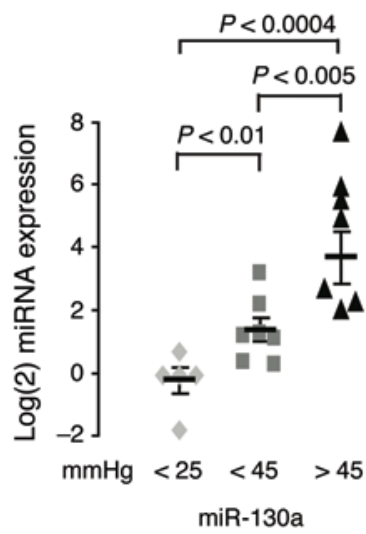

miR-130a probe (blue)
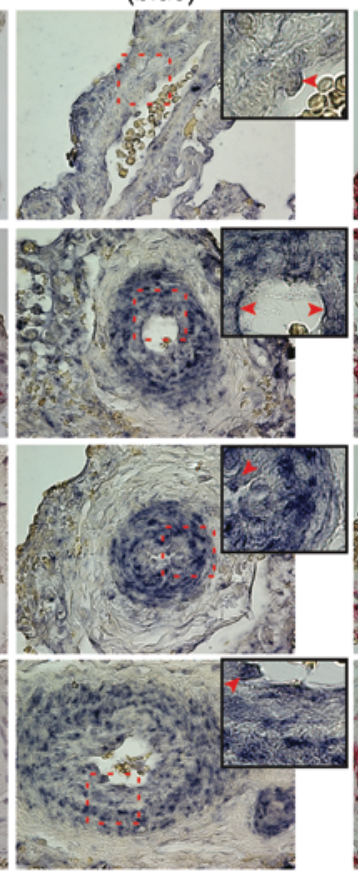

$\alpha$-SMA
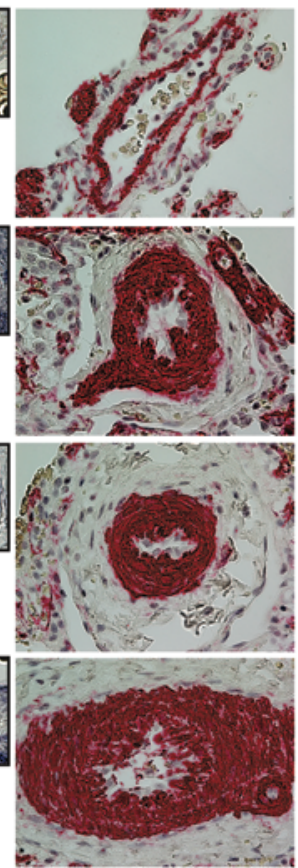

B

Vascular miR-130a expression $\square$ No PAH

PAH

Escleroderma-induced PAH

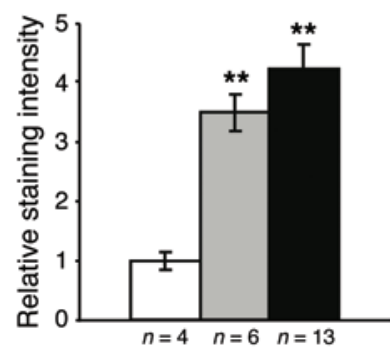

Figure 4. Expression of the miR-130/301 family is upregulated in diverse forms of human PAH. In situ hybridization of human lung (A) and quantification of pulmonary vessels less than $200 \mu \mathrm{m}$ in diameter (10 vessels per patient) (B) revealed increased miR-130a in diseased pulmonary vessels of 6 patients suffering from PAH stemming from a variety of causes as well as in vessels from 13 patients with scleroderma-induced PAH as compared with 4 healthy controls (no PH). By digital zoom (dotted red frame), staining of the intimal and medial layers of the vessel wall was evident (red arrowheads denote intimal layer). See also Supplemental Table 7 for patient demographics. (C) Increasingly higher plasma levels of miR-130/301 family members were observed in patients with increasing hemodynamic severity of $\mathrm{PH}$. Here, 5 non- $\mathrm{PH}$ controls (mPAP $<25 \mathrm{mmHg}$ ) were compared with $\mathrm{PH}$ patients stratified by hemodynamic severity -7 patients with mPAP $25-45 \mathrm{mmHg}$ and 7 patients with mPAP greater than $45 \mathrm{mmHg}$. See also Supplemental Table 8. Statistically significant differences are indicated $\left({ }^{* *} P<0.01\right)$. In the physiologic experiment (C), data are expressed as mean $\pm S E M$, while in the in situ quantitations (B), data are expressed as mean \pm SD. Tissue scale bar: $50 \mu \mathrm{m}$.

sion. Moreover, taken together, these data demonstrated the broad convergence of both genetically associated and acquired PH triggers on coordinated regulation of this key miRNA family.

The miR-130/301 family is upregulated in PH in vivo in animals and humans. Again correlating with our network-based predictions of the broad actions of miR-130/301 in PH, we found consistent upregulation of this miRNA family in whole lung tissue of 8 disparate, yet well-established, animal models of experimental PH (Figure 3, A-C, and Supplemental Figure 3, A-D). These included hypoxia-driven models (mice treated with chronic hypoxia alone, mice treated with chronic hypoxia and the VEGF receptor antagonist SU5416 [ref. 28], and VHL-null mice [ref. 9]); inflammatory-driven models (transgenic IL6 mice [ref. 29], monocrotaline-treated rats, and Schistosoma mansoni-infected mice [ref. 30]); a genetic model of BMPR2 deficiency (transgenic mice overexpressing a dominant-negative mutant BMPR2 [BMPR2X; ref. 31]); and a shunt model of congenital heart disease (juvenile sheep with a surgically placed pulmonary arterial-aortic shunt at late gestation [ref. 32]). In situ staining also revealed increased miR-130a, the most abundantly expressed of the miRNA family 
A
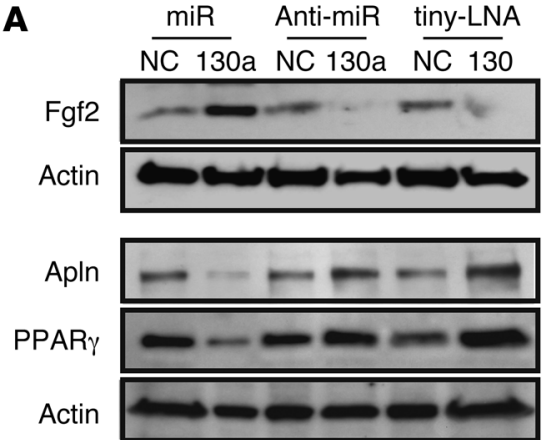

C
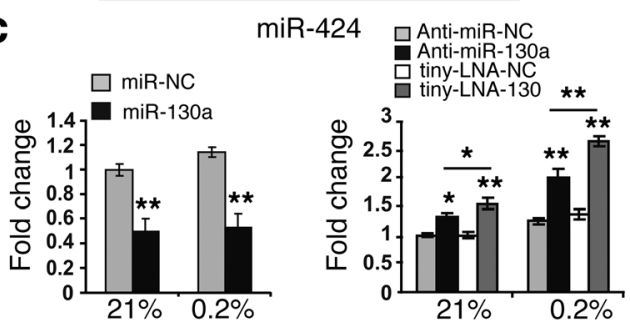

E

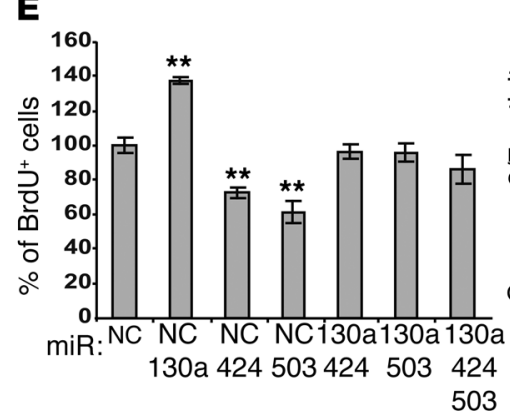

$\mathbf{F}$

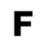

B

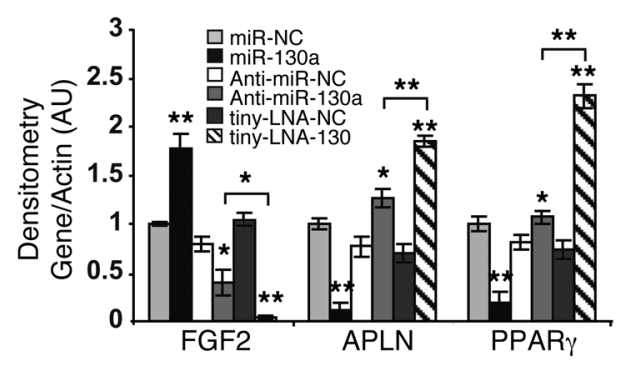

D

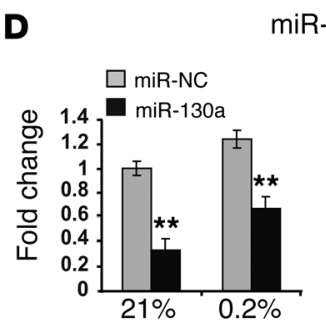

iR-503
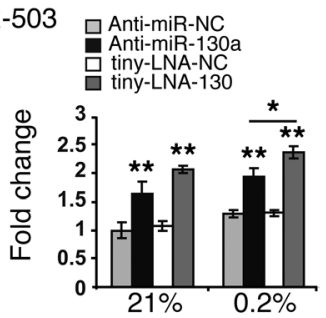

$\mathbf{G}$

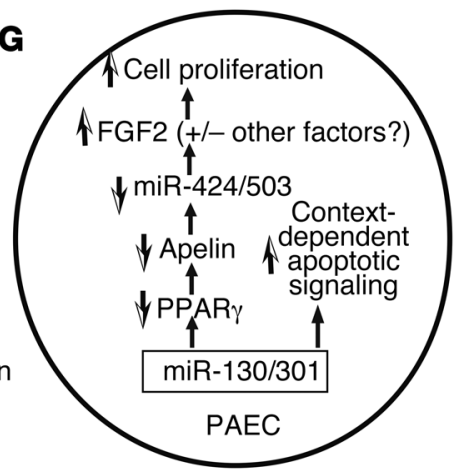

Figure 5. The miR-130/301 family controls PAEC proliferation via the apelin-miR-424/503-FGF2 regulatory axis as well as context-dependent apoptotic signaling. In normoxia $\left(21 \% \mathrm{O}_{2}\right)$ or hypoxia $\left(0.2 \% \mathrm{O}_{2}, 24\right.$ hours), either forced expression of miR-130a mimic versus control (NC) or inhibition of miR-130a (anti-miR-130a) versus inhibition of the entire miR-130/301 family (tiny-LNA-130) versus control (NC) was performed in cultured PAECs (A-D). Immunoblotting (A), gel densitometry (B), and RT-qPCR (C and $\mathbf{D}$ ) revealed that forced miR-130a expression decreased PPAR $\gamma$, a direct target of miR-130/301 (Supplemental Figure 4); decreased apelin (A and B), miR-424 (C), and miR-503 (D); and increased FGF2 (A and B). Inhibition of the entire miR-130/301 family reversed these downstream alterations to a greater extent than miR-130a inhibition alone (A-D), demonstrating the importance of the coordinated actions of this miRNA family. (E) As assessed by BrdU incorporation, PAEC proliferation was augmented by miR-130a mimics but decreased during miR-424 or miR-503 expression. Consistent with the direct dependence of miR-130a on miR-424 and miR-503 in PAECs, miR130a-induced proliferation was reversed when miR-424, miR-503, or both together were expressed. (F) In serum-starved but not serum-replete PAECs, forced expression of miR-130a increased apoptotic caspase 3/7 activity, while miR-130/301 inhibition partially protected cells from caspase $3 / 7$ induction. (C) In PAECs, the miR-130/301 family controls cellular proliferation as well as context-dependent apoptotic signaling. In $\mathbf{C}$ and $\mathbf{D}$, for each miRNA, mean expression in control groups (miR-NC or anti-miR-NC) was assigned a fold change of 1 , to which relevant samples were compared. Data are expressed as mean $\pm \mathrm{SD}\left({ }^{*} P<0.05 ;{ }^{* *} P<0.01\right)$.

members (data not shown), throughout the vascular wall of the small $(<100 \mu \mathrm{m}$ diameter) pulmonary arterioles of mice suffering from PH, induced either alone or by chronic hypoxia plus SU5416 as compared with normoxic mice (Figure 3, D and E). Notably, pulmonary vascular staining intensity for miR-130a linearly correlated with muscular arteriolar wall thickness ( $\alpha$-SMA stain) in these $\mathrm{PH}$ mice, suggesting that at least this miRNA was upregulated in remodeled vascular smooth muscle cells (Figure 3F). To better assess the miR-130/301 expression in vascular endothelial cells in $\mathrm{PH}$, magnetic-activated cell sorting (MACS) was used to separate CD31-positive pulmonary vascular endothelial cells (>95\% purity;
Figure 3G) from digested whole lung tissue of mice. In doing so, we found that such CD31-positive endothelial cells derived from $\mathrm{PH}$ mice (exposed to chronic hypoxia + SU5416) displayed significantly higher levels of miR-130/301 as compared with non-PH control mice (normoxia + SU5416) (Figure $3 \mathrm{H})$. Correlating with these findings in diseased mice, miR-130a expression was also upregulated in both intimal and medial layers of small diseased pulmonary vessels in human lung afflicted with severe forms of $\mathrm{PH}$ (pulmonary arterial hypertension, or PAH) as compared with nondiseased human tissue (Figure 4, A and B; $\mathrm{PAH}$ patient demographics in Supplemental Table 7). Thus, considering the results from both quantitative RT-PCR (RT-qPCR) and in situ staining, miR130/301 expression was upregulated in diseased pulmonary arterioles, affecting both intimal (endothelial) and medial (SMC) layers during $\mathrm{PH}$ in vivo.

Members of the miR130/301 family were also significantly elevated in plasma sampled adjacent to the main pulmonary artery from $\mathrm{PH}$ individuals (mean pulmonary arterial pressure $[\mathrm{mPAP}] \geq 25$ $\mathrm{mmHg}$ ) as compared with non-PH individuals (mPAP $<25 \mathrm{mmHg}$, Figure $4 \mathrm{C}$; patient demographic information in Supplemental Table 8). Further stratification of PH individuals based on pulmonary arterial pressures (e.g., moderate elevation of mPAP between 25 and $45 \mathrm{mmHg}$ as compared with elevation of mPAP $>45 \mathrm{mmHg}$ ) revealed an increasing level of miRNA family member expression with hemodynamic severity. Thus, in animal models and human examples of PH in vivo, expression of the miR-130/301 family is consistently elevated in pulmonary tissue and small diseased pulmonary vessels - a pattern that positively correlates with histologic and hemodynamic severity of disease. 
A

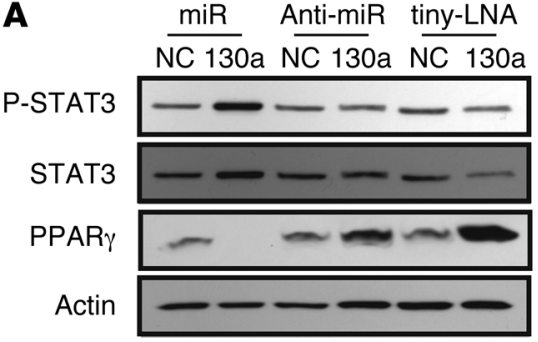

B

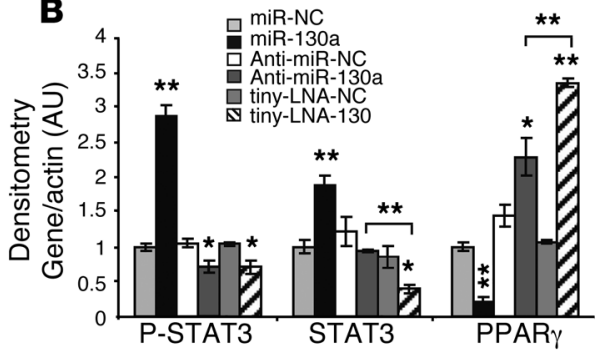

c

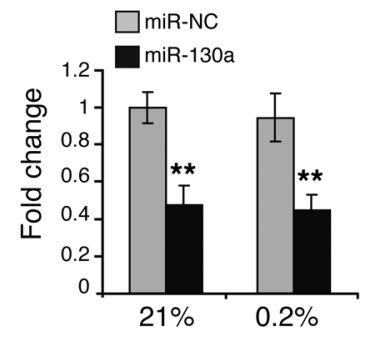

D

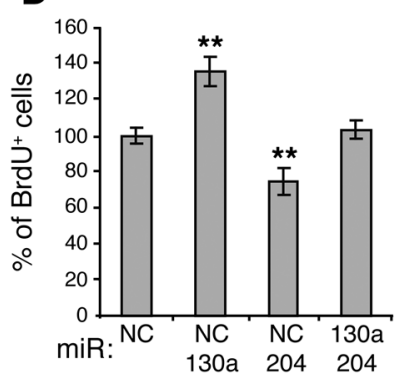

$\operatorname{miR}-204$

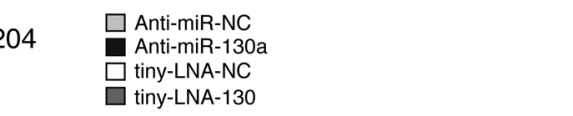

E

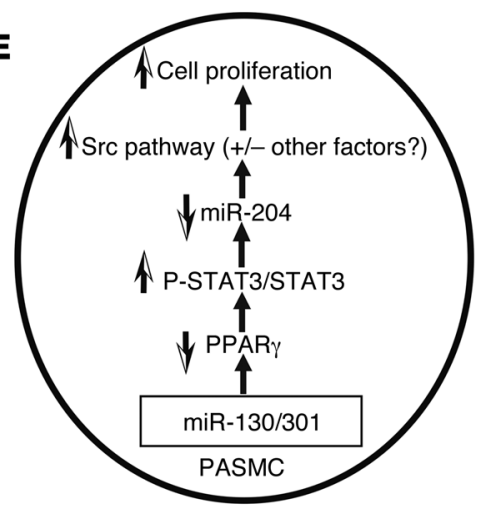

Figure 6. The miR-130/301 family represses PPAR $\gamma$ in order to control proliferation in PASMCs via the STAT3-miR-204 regulatory axis. In normoxia $\left(21 \% \mathrm{O}_{2}\right)$ or hypoxia $\left(0.2 \% \mathrm{O}_{2}, 24\right.$ hours), either forced expression of miR-130a mimic versus control (NC) or inhibition of miR-130a (anti-miR-130a) versus inhibition of the entire miR-130/301 family (tiny-LNA-130) versus control (NC) was performed in cultured PASMCs (A-D). Immunoblotting (A), gel densitometry (B), and RT-qPCR (C) demonstrated that forced miR-130a expression decreased PPAR $\gamma$, accompanied by an increase in STAT3 and activated phosphorylated STAT3 (P-STAT3; A and B), as well as decreased miR-204 (C). Importantly, as in PAECs, inhibition of the entire miR-130/301 family in PASMCs reversed such downstream gene/ subordinate miRNA alterations to a greater extent than inhibition of miR-130a alone (A-D), thus demonstrating the importance of the coordinated actions of this miRNA family. (D) As assessed by BrdU incorporation in exponentially growing PASMCs, proliferation was augmented during forced expression of miR-130a mimics but decreased during expression of miR-204. MiR-130a-induced proliferation was reversed when miR-204 was simultaneously expressed, thus confirming the direct dependence of miR-130a on miR-204 in this cell type. (E) In PASMCs, we have validated that the miR130/301-PPAR $\gamma$ regulatory axis controls proliferation by increasing STAT3 expression and activity and repressing subordinate miR-204 expression. In C, for each miRNA, mean expression in control groups (miR-NC or anti-miR-NC) was assigned a fold change of 1 , to which relevant samples were compared. In all panels, data are expressed as mean \pm SD $\left({ }^{*} P<0.05 ;{ }^{*} P<0.01\right)$

The miR-130/301 family regulates pulmonary vascular cell proliferation via repression of its target PPAR $\gamma$. We next focused our attention on PPAR $\gamma$, a direct target of the miR-130/301 family that was predicted by our network-based approach to mediate the broad actions of this miRNA family specifically in the PH network. Consistent with prior reports in different cellular contexts (33), we confirmed a sequence in the $3^{\prime}$ untranslated region of the PPAR $\gamma$

transcript as a direct binding target for miR-130a (Supplemental Figure 4). Forced expression of miR-130a in PAECs downregulated PPAR $\gamma$ (Figure 5, A and B). Short locked nucleic acid oligonucleotides ("tiny LNAs") with antisense complementarity only to the seed sequence of this miRNA family (tiny-LNA-130; Supplemental Figure 5A and ref. 34) were validated in PAECs and PASMCs as effective inhibitors of the entire miR-130/301 family (Supplemental Figure 5, B and C). Tiny-LNA-130 increased PPAR $\gamma$ expression (Figure 5, A and B), notably to a greater extent than an inhibitor of miR-130a alone. As demonstrated by increased cell number, BrdU incorporation, and increased expression of the proliferation marker PCNA, forced expression of miR-130a increased cell proliferation in both PAECs and PASMCs (Supplemental Figure 6), which was phenocopied by siRNA knockdown of PPAR $\gamma$ (Supplemental Figure 7). Conversely, inhibition of the entire miR-130/301 family was substantially more effective in preventing proliferation than inhibition of miR-130a alone (Supplemental Figure 6). Importantly, forced expression of PPAR $\gamma$ in both cell types reversed the proliferative phenotype induced by miR-130a alone (Supplemental Figure 8). Thus, we confirmed that PPAR $\gamma$ carries an essential role in mediating the proliferative effects of the miR-130/301 family on pulmonary vascular cell types.

The miR-130/301-PPAR $\gamma$ regulatory axis controls context-specific PAEC proliferation and apoptotic signaling by regulating apelin, $m i R-424 / 503$, and FGF2. To further validate our in silico model of the miR-130/301 family, we aimed to determine whether a functional connection truly exists between the miR-130/301 family and the PH-specific pathway connecting apelin, miR-424/503, and FGF2. Because APLN expression is endothelial-specific (Supplemental Figure 14), we reasoned that this regulatory axis may be readily apparent in PAECs. In PAECs, forced expression of miR-130a (or siRNA knockdown of PPAR $\gamma$; Supplemental Figure 10) downregulated apelin, miR424, and miR-503 (Figure 5, A-D, and Supplemental Figure 12A), and upregulated FGF2 (Figure 5, A and B, and Supplemental Figure 12B). Converse regulation of this molecular pathway was observed by inhibition of the miR-130/301 family (Figure 5, A-D, and Supplemental Figure 12) - effects that were again more robust than inhibition of miR-130a alone and effects that were apparent during both normoxia and hypoxia. Demonstrating the essential 
A

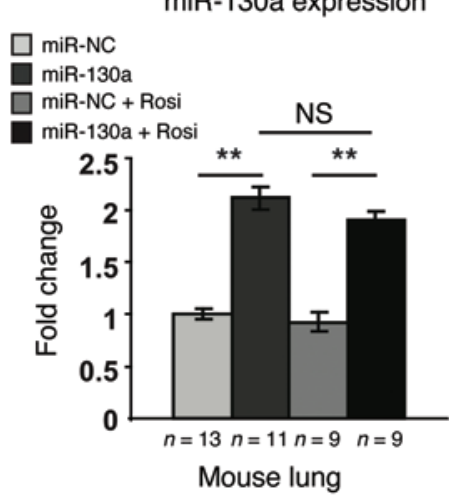

B

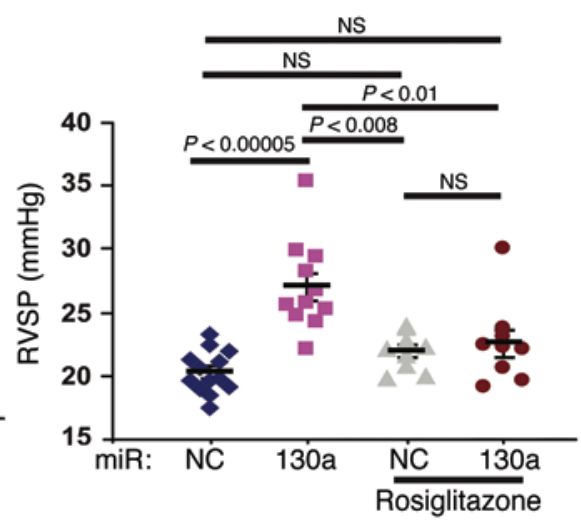

C

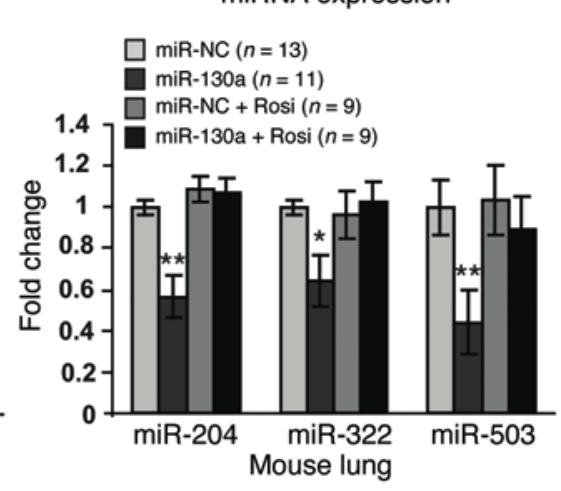

D

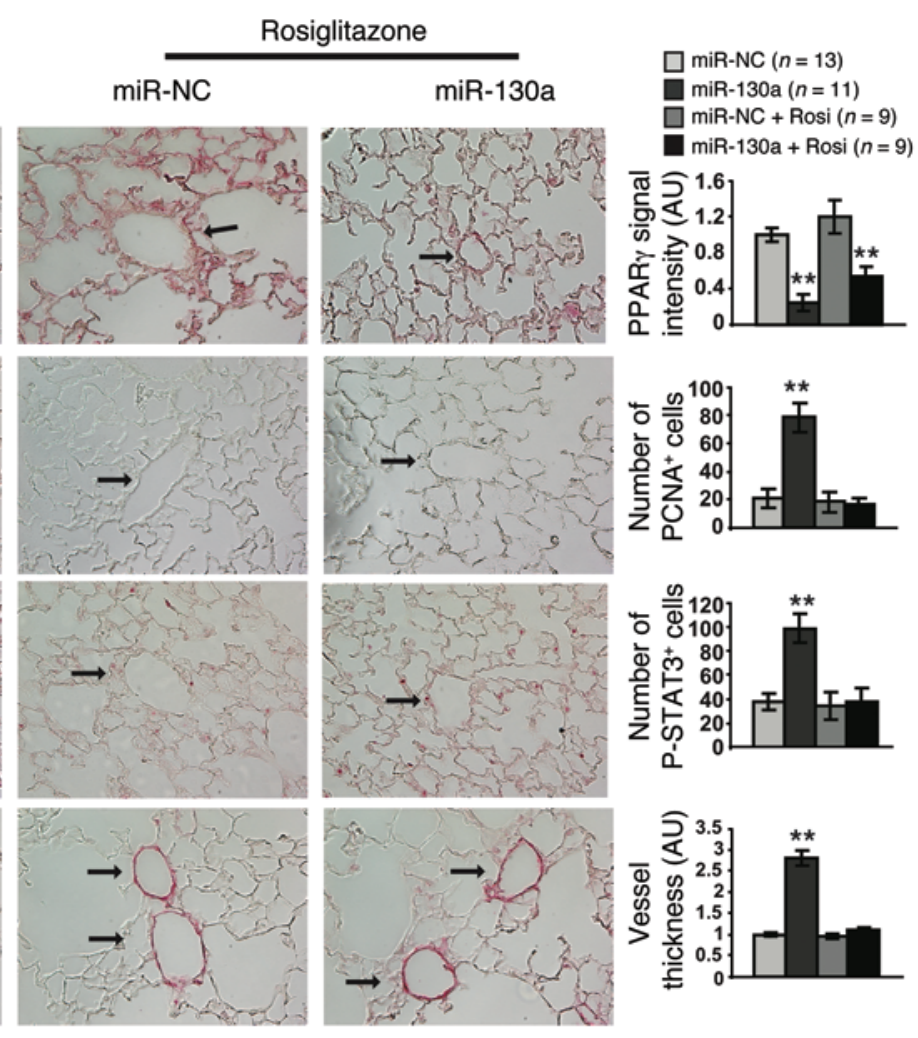

Figure 7. Forced expression of miR-130a induces PH in a PPAR $\gamma$-dependent manner in vivo. (A) Serial intrapharyngeal delivery of miR-130a mimic oligonucleotide (miR-130a) increased miR-130a in whole lung in mice as compared with control (miR-NC), either with or without daily oral dosing of rosiglitazone (Rosi). See Supplemental Figure 18 for protocol design. (B) MiR-130a increased right ventricular systolic pressure (RVSP) compared with control (miR-NC), an effect reversed by rosiglitazone. See Supplemental Figure 21A for quantification of right ventricle/(left ventricle + septum) (RV/LV+S) mass ratio. (C) By RT-qPCR of whole lung, miR-130a decreased miR-204, miR-322 (the mouse homolog of human miR-424), and miR-503 expression, but this effect was reversed by rosiglitazone. (D) IHC staining of pulmonary vessels less than $100 \mu \mathrm{m}$ in diameter was performed to quantify PPAR $\gamma$, PCNA, phosphorylated STAT3 (P-STAT3), and $\alpha$-SMA. Ten vessels were quantified for each mouse (bar graphs). Consistent with data from cultured cells (Figures 5 And 6), miR-130a decreased PPAR $\gamma$ expression (top row); increased PCNA-positive (second row) and P-STAT3-positive (third row) cells (quantified over 10 vessels); and increased medial thickness (bottom row) consistent with exaggerated vascular remodeling. See Supplemental Figure 21B for arteriolar density, Supplemental Figure 21C for arteriolar muscularization, and Supplemental Figure 21, D and E, for gene expression by immunoblotting. In A and C, for each miRNA, mean expression in control groups (miR-NC) was assigned a fold change of 1, to which relevant samples were compared. In experiments involving animal tissue $(\mathbf{A}-\mathbf{C})$, data are expressed as mean \pm SEM, while IHC quantification (D) is expressed as mean \pm SD $\left({ }^{*} P<0.05\right.$; $\left.{ }^{*} P<0.01\right)$. Scale bar: $50 \mu$ m.

role of PPAR $\gamma$ in these responses, miR-130a-dependent gene alterations were rescued by restoration of PPAR $\gamma$ function by either forced PPAR $\gamma$ expression (Supplemental Figure 13, A-D) or pharmacologic PPAR $\gamma$ activation (rosiglitazone) (Supplemental Figure $13, \mathrm{E}-\mathrm{H})$. Notably, we found that a number of PPAR $\gamma$-dependent target genes related to the cell cycle, such as CCND1, CDKN1A, $C D K N 2 A$, and $C D K N 2 B(19,35-37)$, were also modulated by manipulation of the miR-130/301 family or PPAR $\gamma$ (Supplemental Figure 11, A-D). However, proving the critical role of miR-424/503 in this regulatory network, forced expression of miR-424 or miR- 
A miR-130/301 family expression

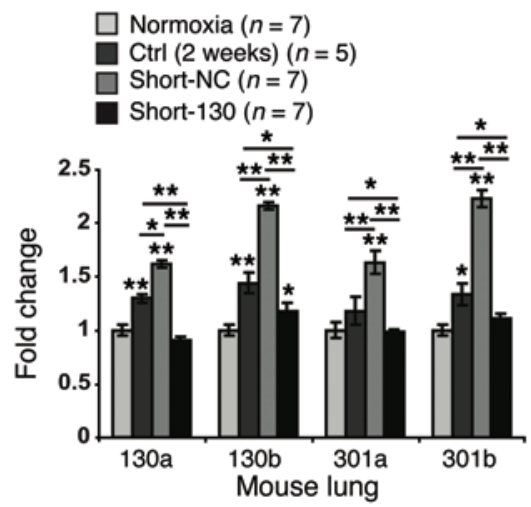

D
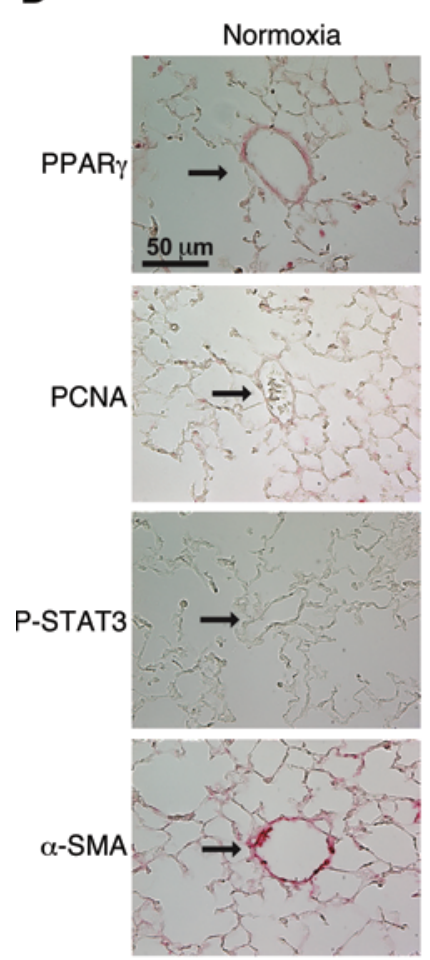

B

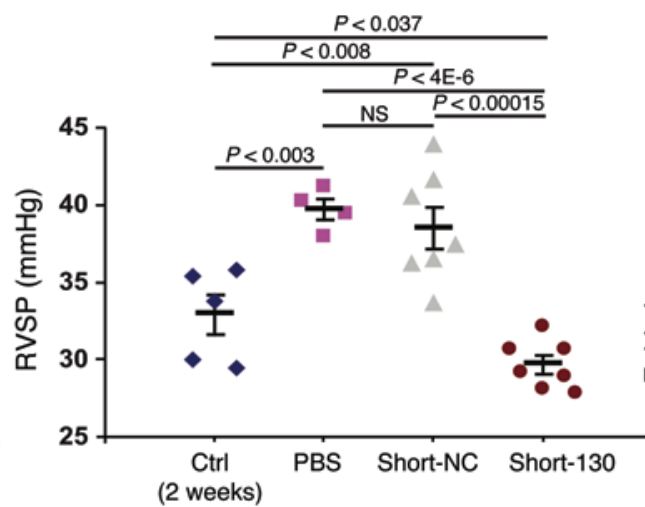

Hypoxia
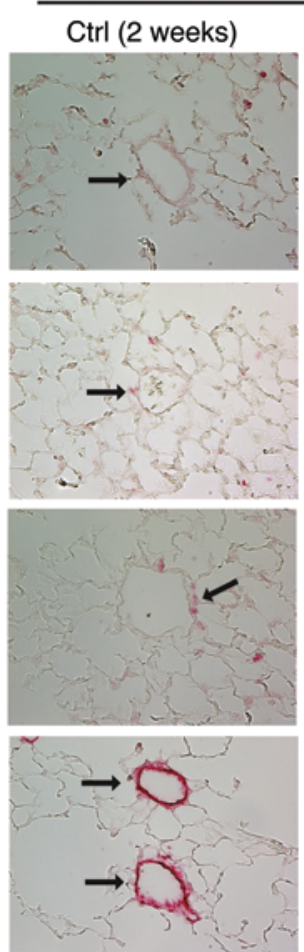

Short-NC
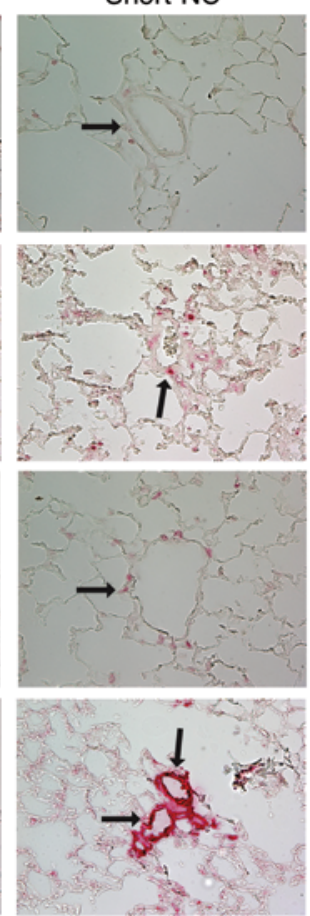

C

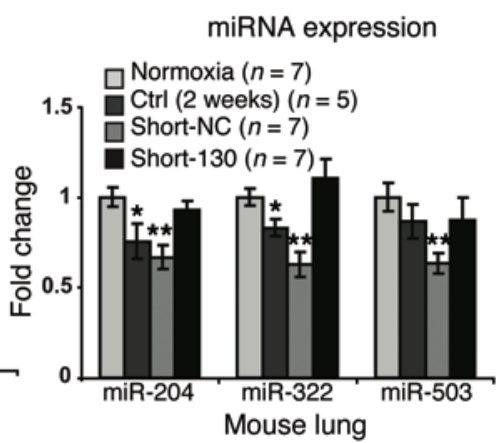

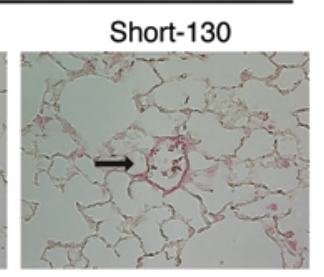

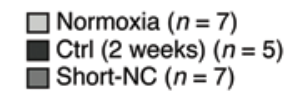

$\square$ Short-130 (n=7)
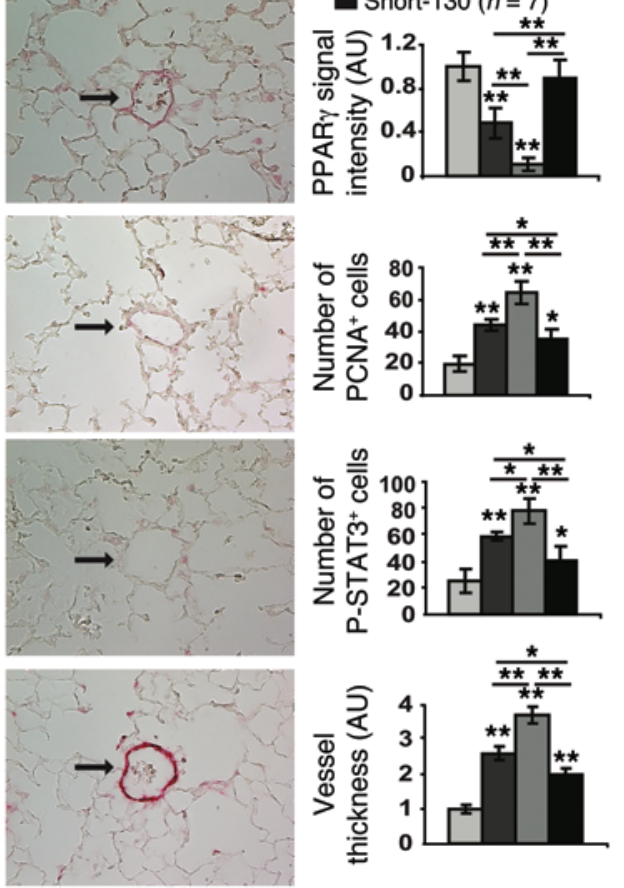

Figure 8. The miR-130/301 family is necessary to induce PH in a hypoxic mouse model of disease. A protocol was performed to determine whether a "shortmer" inhibitor of miR-130/301 (short-130) reverses PH (hypoxia + SU5416) in mice. See Supplemental Figure 23. (A) While miR-130/301 progressively increased with exposure time to hypoxia + SU5416, short-130 maintained baseline miRNA expression (normoxia + SU5416). (B) RVSP progressively increased as mice were exposed to 2 weeks (Ctrl 2 weeks) and 4 weeks of hypoxia + SU5416 (PBS). Increased RVSP was abrogated by short-130 but not short-NC. See Supplemental Figure 26A for quantification of (RV/LV+S) mass ratio. (C) During hypoxia + SU5416, short-130 rescued miR-204, miR-322 (mouse homolog of human miR-424), and miR-503 expression in whole lung. (D) IHC staining of pulmonary vessels less than $100 \mu$ m diameter was performed to quantify PPAR $\gamma$, PCNA, phosphorylated STAT3 (P-STAT3), and $\alpha$-SMA. Ten vessels were quantified for each mouse (bar graphs). Inhibition of the miR-130/301 family after PH development preserved PPAR $\gamma$ expression (top row); decreased PCNA-positive (second row) and P-STAT3-positive (third row) cells (quantified over 10 vessels); and decreased medial thickness (bottom row) consistent with blunted vascular remodeling. See Supplemental Figure 26B for arteriolar density, Supplemental Figure $26 \mathbf{C}$ for arteriolar muscularization, and Supplemental Figure 26, D and E, for immunoblotting. In A and C, for each miRNA, normoxic levels were assigned a fold change of 1 , to which relevant samples were compared. Data involving animal tissue (A-C) are expressed as mean \pm SEM, while IHC quantification (D) is expressed as mean $\pm \mathrm{SD}\left({ }^{*} P<0.05\right.$; $\left.{ }^{* *} P<0.01\right)$. Scale bar: $50 \mu \mathrm{m}$.

503 individually - and to a better extent when both miRNAs were expressed together - reversed the proliferative response to miR130a (Figure 5E). Thus, in PAECs, the miR-130/301-PPAR $\gamma$ regulatory axis controls cellular proliferation by repressing apelin and its subordinate miRNAs (miR-424/503; Figure 5G), thus validating one arm of the $\mathrm{PH}$-specific hierarchy of miRNA regulation predicted by network analysis.
Beyond the network-based predictions regarding endothelial proliferation, we found that forced expression of miR-130a increased apoptotic caspase signaling in serum-starved cultured PAECs, while inhibition of miR-130/301 protected against these effects (Figure 5F). These observations are consistent with prior observations of reduced APLN causing endothelial apoptosis (38) as an inciting pathogenic event to allow for selection of apoptosis- 


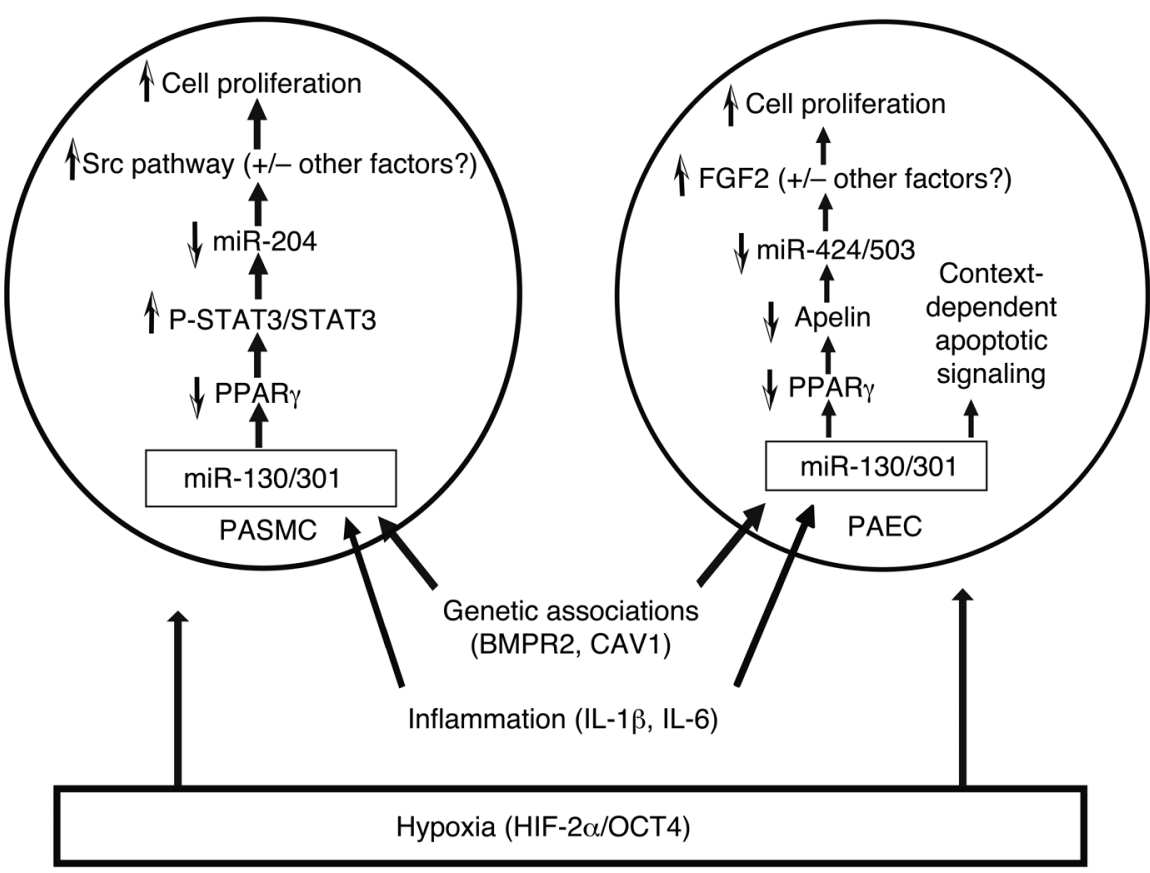

Figure 9. Model of the actions of the miR-130/301 family in PH. Network-based bioinformatics coupled with experimental validation identifies the broad hierarchical control over proliferation exerted by the miR-130/301 family via integration of numerous subordinate miRNA pathways in multiple pulmonary vascular cell types.

erative response of PASMCs to miR-130a (Figure 6D). Thus, validating the second arm of network predictions in PASMCs, the miR-130/301-PPAR $\gamma$ regulatory axis controls proliferation primarily by repressing STAT3 expression and activity and subordinate miR-204 expression (Figure 6E).

The miR-130/301 family controls PPAR $\gamma$ and subordinate miRNA pathways to promote $\mathrm{PH}$ in vivo. Based on the integrated functions of the miR-130/301 family as delineated above, we wanted to determine whether chronic miR-130/301 induction is necessary and sufficient to promote $\mathrm{PH}$. To maintain consistency in these experiments,

resistant PAECs in PAH (39). Notably, however, this miRNA family did not exert similar control over apoptotic activity in serumstarved PASMCs (Supplemental Figure 9), nor were these actions evident in the presence of serum (Supplemental Figure 9). Thus, this dual nature of miR-130/301 promoting context-specific apoptotic signaling and proliferation in PAECs indicates both the complexity of, and the substantially broad control over, multiple disparate pathways that converge to promote $\mathrm{PH}$ (Figure $5 \mathrm{G}$ ).

The miR-130/301-PPAR $\gamma$ axis controls PASMC proliferation via regulation of miR-204 and STAT3. We then interrogated the functional relationship between the miR-130/301 family and the STAT3-miR-204-Src kinase pathway, as predicted by our networkbased modeling. Given prior reports that PPAR $\gamma$ represses STAT3 transcription (40) and the localized actions of miR-204 in PASMCs (7), we further hypothesized that, in PASMCs, miR-130/301-dependent repression of PPAR $\gamma$ upregulates STAT3 expression and/or activity and thus downregulates miR-204. Correspondingly, in PASMCs, forced expression of miR-130a (or siRNA knockdown of PPAR $\gamma$; Supplemental Figure 15) decreased PPAR $\gamma$, accompanied by increased STAT3 expression and decreased miR-204 (Figure 6, A-C, and Supplemental Figure 12C). As in PAECs, these expression changes were reversed by either forced expression of PPAR $\gamma$ (Supplemental Figure 16, A-C) or rosiglitazone (Supplemental Figure 16, D-F), and converse regulation of this molecular pathway was observed during normoxia and hypoxia by inhibition of the miR-130/301 family but not miR-130a alone (Figure 6, A-C). Notably, we analyzed other downstream targets of STAT3 such as NFATC2 and PIM1 (Supplemental Figure 17), known to be important in proliferation $(7,41)$ as well as other $\mathrm{PH}$-relevant phenotypes (42). Manipulation of either miR-130/301 or PPAR $\gamma$ modulated these target genes, suggesting a broad impact of the miR-130/301PPAR $\gamma$-STAT3 axis in control of PASMC-specific phenotypes beyond proliferation alone. Nonetheless, as with miR-424/503 in PAECs, forced miR-204 expression prevented the specific prolifa mouse model of PH was employed via treatment with SU5416 and chronic hypoxia (28). First, in the presence of SU5416, chronic pulmonary expression of miR-130a in WT mice was studied in place of chronic hypoxia. MiRNA delivery was achieved by 4 serial weekly intrapharyngeal injections of liposomally encapsulated miR-130a oligonucleotide mimics, as adapted from prior protocols (ref. 7 and Supplemental Figure 18). Such delivery resulted in upregulation of miR-130a (but not other family members) in whole lung tissue (Figure 7A and Supplemental Figure 19A) and in small pulmonary vessels (Supplemental Figure 19B). This delivery also repressed expression of pulmonary vascular PPAR $\gamma$ (as quantified by immunohistochemistry [IHC] methods as previously described in ref. 9 and Figure 7D and by immunoblot in Supplemental Figure 21, D and E). Notably, such local delivery confined expression changes predominantly to the lung (Supplemental Figure 19C), without alteration in left ventricular function as assessed by echocardiography (Supplemental Figure 20). In turn, miR-130a expression upregulated pulmonary vascular STAT3 phosphorylation (IHC, Figure 7D; and immunoblot, Supplemental Figure 21, D and E) and repressed miR-204, miR-322 (the homolog of miR-424 in mice), and miR-503 expression (Figure 7C), thus leading to vascular proliferation as reflected by PCNA labeling (Figure 7D). As a result, chronic miR-130a expression increased pulmonary vascular remodeling as demonstrated by increased medial thickness in small $(<100 \mu \mathrm{m}$ diameter) pulmonary arterioles ( $\alpha$-SMA stain; Figure 7D), an increased percentage of muscularized arterioles (Supplemental Figure 21C), and a decreased number of small pulmonary arterioles (Supplemental Figure 21B). In turn, pulmonary vascular expression of miR-130a significantly increased right ventricular remodeling (increased right ventricle/(left ventricle + septum) $[\mathrm{RV} / \mathrm{LV}+\mathrm{S}]$ mass ratio; Supplemental Figure 21A) and right ventricular systolic pressure (RVSP), a hemodynamic surrogate for pulmonary arterial pressure (Figure 7B). Importantly, similar PH manifestations were observed in mice treated with miR-130a without SU5416 (Supplemental Figure 22). 
Notably, miR-130a-dependent gene expression changes as well as most histologic and hemodynamic alterations (Figure 7 and Supplemental Figure 21) were reversed by rosiglitazone, thus demonstrating the necessary role of PPAR $\gamma$ for these pathogenic events in vivo. Thus, miR-130a expression depends, at least in part, on repression of PPAR $\gamma$ to control a predicted hierarchy of subordinate miRNA pathways in vivo in order to promote pulmonary vascular disease.

To determine whether miR-130/301 is necessary for manifestation of $\mathrm{PH}$ under hypoxic stimuli, inhibition of this miRNA family in the pulmonary vasculature was achieved by pharmacologic means. After 2 weeks of SU5416 and chronic hypoxia, PH was confirmed in mice by RVSP elevation (Figure 8B) as well as right ventricular remodeling (Supplemental Figure 26A) and pulmonary arteriolar remodeling (Figure 8D and Supplemental Figure 26, B and C). This treatment was followed by 2 additional weeks of hypoxia plus SU5416 in the setting of 3 serial intrapharyngeal injections of either a chemically modified, short anti-miRNA ("shortmer") complementary to the seed sequence or a scrambled control (Supplemental Figure 23). Shortmer delivery was confirmed specifically in pulmonary tissue as assessed by repression of the miR-130/301 family members to baseline, nondiseased conditions in whole lung by RT-qPCR (Figure 8A), in lung vasculature by IHC staining of the shortmer backbone (Supplemental Figure 24B), and in lung vasculature by visualization of a fluorescent Cy5-labeled shortmer (Supplemental Figure 24A). Consistent with our mode of intrapharyngeal delivery, such shortmer expression was not seen in other organs beyond the lung (Supplemental Figure 24A), including the left ventricle where function was unaffected with such administration (Supplemental Figure 25). In direct correlation with our mechanistic findings defined in cultured PAECs and PASMCs (Figures 5 And 6), during disease provocation, inhibition of the miR-130/301 family (with Short-130) derepressed pulmonary vascular PPAR $\gamma$ expression in order to decrease STAT3 phosphorylation (by IHC [Figure 8D] and immunoblot [Supplemental Figure 26, D and E]), increase expression of its subordinate miRNAs miR-204, miR-424, and miR-503 (Figure 8C), and decrease vascular proliferation as reflected by PCNA expression (Figure 8D). Importantly, such molecular events markedly reduced pulmonary vascular remodeling (reflected by medial thickness [Figure 8D] and percentage of muscularized arterioles [Supplemental Figure 26C] after IHC stain for $\alpha$-SMA), loss of small pulmonary arterioles (Supplemental Figure 26B), right ventricular remodeling (Supplemental Figure 26A), and RVSP (Figure 8B), with a trend toward levels even lower than at the starting point of disease (2-week time point). Thus, considering both gain-of-function and loss-of-function experimentation, we conclude that chronic induction of endogenous miR-130/301 family members is necessary and sufficient to promote $\mathrm{PH}$ in vivo, and such robust actions depend on hierarchical control of PPAR $\gamma$ and subordinate miRNAs, as predicted by our network-based analyses.

\section{Discussion}

In this study, driven by an advanced analysis of the molecular network architecture specific for $\mathrm{PH}$, we identified unique systemslevel regulation of this disease by miR-130/301, orchestrating subordinate miRNA and target gene networks to control cellular proliferation and $\mathrm{PH}$ manifestation in vivo. Importantly, the suppression of miR-204 and miR-424/503 by the miR-130/301 family represents the first description to our knowledge of a functional hierarchy of seemingly disparate miRNAs relevant to PH. Furthermore, we found that such broad control extends not only to multiple signaling pathways but also across disparate cell types. Thus, these data (Figure 9) emphasize that a single set of upstream master miRNAs integrates the control of pulmonary vascular crosstalk and $\mathrm{PH}$-relevant phenotypes via interconnected mechanisms that had previously been missed by conventional discovery strategies. Accordingly, such findings have important implications for our fundamental understanding of the upstream origins of $\mathrm{PH}$, for improvements in diagnostic and therapeutic applications in this disease, and for the increased use of in silico network theory to decipher additional systems-level regulatory mechanisms of miRNAs in complex disease networks.

These findings provide critical validation for the evolving application of network theory to the discovery of the combinatorial origins of $\mathrm{PH}$ and other diseases. To date, systems biology approaches have been developed to elucidate the higher-order regulation of human disease gene networks mainly in singlecell contexts amenable to high-throughput profiling, such as in cancer (43). However, such profiling has not been possible in diseases such as $\mathrm{PH}$, where diseased tissue is challenging to obtain and pathogenesis spans multiple heterogeneous cell types (as we review in ref. 44). Consequently, substantial concern has been raised regarding the ability to discern the key factors - miRNAs or otherwise - controlling $\mathrm{PH}$ phenotypes among the exponentially increasing interconnections being mapped among PH-relevant genes, effectors, and cell types. On the other hand, we and others have proposed computational analyses of the overarching architecture of existing integrated molecular disease networks to bypass some of these obstacles (as reviewed by ref. 10). However, in vivo validation of these theories had been limited until now. Here, primarily based on statistical analyses of available network architecture, our use of network theory and experimental biology indeed predicts and confirms an expansive miRNA-based disease network in $\mathrm{PH}$ and supports the notion that available molecular maps in $\mathrm{PH}$ are adequate for such modeling. Mapping of the complete disease interactome would be ideal, but anatomic inaccessibility of pulmonary vascular cell types in vivo and financial infeasibility for extensive measurements limit such data in PH (45). The level of overdetermination and redundancy in complex biologic networks (10) may be a key factor in allowing us to perceive such higher-order regulation even if some genes or pathways are missing from the current interactome maps. Consequently, future endeavors to map similar miRNA-target networks in $\mathrm{PH}$ and other diseases hold great promise for rapidly identifying other master regulators otherwise hidden in the architecture of existing disease networks.

The actions of the miR-130/301 family members as a collective rather than reliance on 1 individual miRNA may underlie their combinatorial robustness of action in $\mathrm{PH}$. The cohesive upregulation of the miR-130/301 family as a unit despite separate chromosomal locations reflects the evolutionary selection pressure to coordinate their actions. The importance of their simultaneous actions is further emphasized by the intricate yet distinct methods by which these miRNAs are induced under various $\mathrm{PH}$ triggers. These include an NF- $\mathrm{BB}$ dependence during inflammatory 
cytokine stimulation (13), a dependence on BMPR2 (Supplemental Figure $1 \mathrm{C}$ and Supplemental Figure 3C) and CAV1 (Supplemental Figure 1), and a unique HIF-2 $\alpha$-POU5F1/OCT4 dependence during hypoxia (Figure 2). The intriguing dependence of miR-130/301 expression on BMPR2 and CAV1 sets the stage for deeper mechanistic interrogation, perhaps regarding whether endogenous mutations in both of these genes are functionally linked to PAH via this miRNA family. Furthermore, given the previously described association of $\mathrm{PH}$ with a gain-of-function mutation of HIF- $2 \alpha$ seen in people with Chuvash polycythemia (46), it is tempting to speculate the importance of the miR-130/301 family in the pathobiology of this genetic predisposition as well. Pathogenic alterations of other unidentified regulatory factors may upregulate this miRNA family in the context of other PH triggers. Intriguing possibilities may include dehydroepiandrosterone (DHEA) (47) or the DNA damage effector poly-(ADP-ribose) polymerase-1 (PARP-1) (48), both of which influence the STAT3-miR-20 4 axis in PH. Ultimately, such convergent biology (Figure 1) could reflect synergism among disparate stimuli to upregulate miR-130/301 more robustly during $\mathrm{PH}$ progression - actions that may be best delineated through further network-based bioinformatics analyses. To coordinate miRNA function, principles of redundancy and synergy may also figure prominently but have been poorly defined in vivo, especially in PH. For the miR-130/301 family, which shares the same "seed sequence," our primary data emphasize the biologic importance of target binding redundancy to allow for the maintenance of miRNA action without relying on 1 factor alone. Such results also highlight the importance of strategic inhibition of multiple related miRNAs for maximum therapeutic effect. Finally, future work can be envisioned to interrogate the synergy of action of multiple miR-130/301 family members that bind the same target with varying efficiencies (typically determined by "non-seed sequences") and may depend on coordinated effects on miRNA binding and mRNA structure to maximize target gene repression.

Importantly, while this report focuses mainly on proliferative actions of miR-130/301, our experiments validated only a small minority of the network predictions. Thus, the true breadth of influence by the miR-130/301 family in PH may be even more extensive. Specifically, our findings expand on the importance of PPAR $\gamma$ in PH $(19-21,24,49)$, revealing a more complete spectrum of its miRNA-dependent functions in the pulmonary vasculature. The known pleiotropy of PPAR $\gamma$ influencing vasoconstriction, metabolism, and ECM deposition in the peripheral vasculature and as suggested by our work examining additional PPAR $\gamma$ targets responsive to miR-130/301 (Supplemental Figure 11) further suggests that this miRNA family controls even more $\mathrm{PH}$-specific phenotypes than elucidated here. Moreover, our data should prompt future work to determine a more detailed molecular explanation of how the miR-130/301-PPAR $\gamma$ axis controls both context-specific apoptotic signaling (Supplemental Figure 9) and proliferation (Figure 5) in PAECs in order to converge on promoting PH. Beyond PPAR $\gamma$, additional miR-130/301 targets may regulate a variety of $\mathrm{PH}-$ relevant pathways including TGF-BMP signaling, Rho kinase activation, vascular smooth muscle contraction, and mitochondrial metabolism, among others (Figure 1C). Moreover, beyond the miR-130/301 family, both subordinate miRNAs miR-424 and miR204 themselves were predicted in the top 10 miRNAs by miRNA spanning score as comparably broad regulators of the expanded PH network (Supplemental Table 2). Taken together, convergent regulation of all of these factors by the miR-130/301 family may offer additional layers of cooperativity and ultimately may allow for both more precise and more robust control over disease manifestation than most previously characterized $\mathrm{PH}$ disease genes.

A molecular appreciation of the systems-level actions of the miR-130/301 family could also aid the development of more effective clinical management strategies for PH. From a diagnostic angle, expanding on prior studies (50), we found that expression of the miR-130/301 family in pulmonary arterial plasma increased with worsening hemodynamic severity of PH (Figure 4C). Coupled with the related functions of these miRNAs in $\mathrm{PH}$, such results provide a foundation for a larger study aimed at characterizing this miRNA family as a profile of $\mathrm{PH}$ biomarkers that could offer more specificity as compared with a single miRNA alone. From a therapeutic angle, the unique application of shortmer technology (34) to pulmonary vascular disease in vivo could impact substantially the rational treatment of $\mathrm{PH}$ by inhibiting multiple, but related, miRNAs for greater amelioration of disease. Moreover, a rational combination of pharmacologic PPAR $\gamma$ activation along with repression of this miRNA family could have even synergistic effects on protection and reversal of this disease. Consequently, we envision that further experimental validation of miRNA network architecture may have a dramatic impact on systems pharmacology approaches in $\mathrm{PH}$ that otherwise have not yet been pursued in great depth.

In summary, through advanced analysis and validation of disease network architecture, we have defined a higher order of miRNA network regulation in $\mathrm{PH}$ by the miR-130/301 family, thus addressing a notable deficiency in reductionist experimentation and carrying broad implications for miRNA-based diagnostics and therapeutics. Consequently, future applications of miRNA network theory should rapidly define additional upstream origins of $\mathrm{PH}$ and perhaps other disease conditions that link complex miRNA signaling pathways to final disease manifestations.

\section{Methods}

Network design. A complete set of methods to construct the initial curated and expanded $\mathrm{PH}$ networks and to perform spectral partitionbased clustering of those networks is available online (Supplemental Methods). The Supplemental Methods also contain detailed methods for analysis of network architecture, including calculations for the miRNA spanning score, target spanning score, gene spanning score, and shared miRNA influence score.

Oligonucleotides and transfection. Pre-miRNA oligonucleotides (pre-miR-130a, pre-miR-204, pre-miR-424, and pre-miR-503 and negative control pre-miR-NC1 and pre-miR-NC2, Life Technologies), anti-miRNA oligonucleotides (anti-miR-130a and anti-miR-NC, ThermoScientific), tiny LNA oligonucleotides (tiny-130, 5'-ATTGCACT-3', and tiny-NC, 5'-TCATACTA-3', Exiqon), and siRNAs (PPAR $\gamma$ and scrambled control, Santa Cruz Biotechnology; HIF- $1 \alpha$, HIF- $2 \alpha$, and scrambled control, Life Technologies) were commercially purchased. PAECs and PASMCs were plated and transfected 24 hours later at 70\%-80\% confluence using pre-miRNA ( $5 \mathrm{nM})$, anti-miRNA (20 nM), tiny-LNA (20 nM), or siRNA ( $25 \mathrm{nM})$ with Lipofectamine 2000 reagent (Life Technologies), according to the manufacturer's instructions. 
Forced expression of miR-130a in mouse lung. Eight-week-old mice (C57BL/6) were injected with SU5416 weekly $(20 \mathrm{mg} / \mathrm{kg} / \mathrm{dose}$; Sigma-Aldrich), accompanied by 4 intrapharyngeal injections (once per week) of $1 \mathrm{nmol}$ of miR-control (pre-miR-NC) or miR-130a (pre-miR130a) mixed in $100 \mu \mathrm{l}$ PBS solution containing 5\% Lipofectamine 2000 (Life Technologies). Three days after the last injection, right heart catheterization was performed as previously described (9), followed by harvesting of lung tissue for RNA extraction or paraffin embedding, as described above. Rosiglitazone (Cayman Chemicals) was dissolved in $0.25 \%$ carboxymethyl cellulose medium-viscosity aqueous solution. This rosiglitazone solution versus vehicle control was delivered daily $(20 \mathrm{mg} / \mathrm{kg} / \mathrm{d}$ ) by oral gavage to mice for 3 weeks.

Inhibition of the miR-130/301 family in mouse lung. Eight-weekold mice (C57BL/6) were injected with SU5416 $(20 \mathrm{mg} / \mathrm{kg} / \mathrm{dose}$; Sigma-Aldrich), followed by exposure to normobaric hypoxia (10\% $\mathrm{O}_{2}$; OxyCycler chamber, Biospherix Ltd.) for 2 weeks. After 2 weeks and confirmation of $\mathrm{PH}$ development in 5 mice (right heart catheterization), mice were further treated with 3 intrapharyngeal injections (every 4 days) of control or miR-130/301 shortmer oligonucleotides, designed as fully modified antisense oligonucleotides complementary to the seed sequence of the miR-130/301 miRNA family $(10 \mathrm{mg} /$ $\mathrm{kg}$ /dose; Regulus). Specifically, the control and miR-130/301 shortmer oligonucleotides were nontoxic, lipid-permeable, high-affinity oligonucleotides. The miR-130/301 shortmer carried a sequence complementary to the active site of the miR-130/301 miRNA family, containing a phosphorothioate backbone and modifications (fluoro-, methoxyethyl, and bicyclic sugar) at the sugar 2' position. Three days after the last injection, right heart catheterization was performed followed by harvesting of lung tissue for RNA extraction or paraffin embedding, as described above.

Statistics. Cell culture experiments were performed at least 3 times and at least in triplicate for each replicate. The number of animals in each group was calculated to measure at least a $20 \%$ difference between the means of experimental and control groups with a power of $80 \%$ and SD of $10 \%$. The number of unique patient samples for this study was determined primarily by clinical availability. RT-qPCR on human plasma, in situ expression/histologic analyses of both mouse and human tissue, and pulmonary vascular hemodynamics in mice were performed in a blinded fashion. Numerical quantifications for in vitro experiments using cultured cells or in situ quantifications of transcript/miRNA expression represent mean \pm SD. Numerical quantifications for physiologic experiments using mice or human reagents represent mean \pm SEM. Immunoblot images are representative of experiments that were repeated at least 3 times. Micrographs are representative of experiments in each relevant cohort of mice. Paired samples were compared by Student's $t$ test (2-tailed). Comparison of multiple samples was performed by ANOVA followed by Student Newman-Keuls post hoc testing. Correlation analyses were performed by Pearson correlation coefficient calculation, as previously described (8). A $P$ value less than 0.05 was considered significant.

Study approval. All animal experiments were approved by the Harvard Center for Comparative Medicine and the Committees on Animal Research of the University of California, San Francisco, University of Colorado, Denver, and Vanderbilt School of Medicine. All experimental procedures involving the use of human tissue and plasma were approved by the Partners HealthCare, Boston Children's Hospital, and University of California, Los Angeles, Institutional Review Boards and the New England Organ Bank. Ethical approval for this study conformed to the standards of the Declaration of Helsinki. Informed consent was obtained for right heart catheterization and blood sampling. For formalin-fixed paraffin-embedded lung samples, human $\mathrm{PH}$ specimens were collected from unused or discarded surgical samples; nondiseased human lung specimens from the New England Organ Bank have been described (9). For plasma harvesting and analysis, human subjects were chosen with clinically significant dyspnea and undergoing right heart catheterization. Subjects were stratified by the presence or absence of clinical PH, as defined by elevated $\mathrm{mPAP} \geq 25 \mathrm{mmHg}$.

Additional information. In the Supplemental Methods online, previously published procedures are described for cell culture, plasmid construction, in silico transcription factor binding analyses, lentivirus production, luciferase reporter assay validation of miRNA targets, BrdU proliferation assays, caspase $3 / 7$ apoptosis assay, plasma and cellular RNA extraction, RT-qPCR, immunoblotting, tissue harvesting, in situ miRNA staining, IHC, pulmonary artery quantification, and fluorescence microscopy (9). Details regarding published animal models of $\mathrm{PH}$, isolation of mouse pulmonary vascular endothelial cells, and human plasma sampling are also found there.

\section{Acknowledgments}

We thank J. Loscalzo, J. Snow, B. North, S.K. Chan, and R. Wang for critical review of the manuscript and S. Tribuna for expert administrative assistance. We thank A.-L. Barabasi for provision of the consolidated interactome database. This work was supported by the NIH (grants K08-HL096834 to S.Y. Chan, HL67841 to S.M. Black, HL61284 to J.R. Fineman); the McArthur-Radovsky, Lerner, Harris, and Watkins Funds; and the Pulmonary Hypertension Association (to S.Y. Chan).

Address correspondence to: Stephen Y. Chan, Brigham and Women's Hospital, New Research Building, Room 630N, 77 Avenue Louis Pasteur, Boston, Massachusetts 02115, USA. Phone: 617.525.4844; E-mail: sychan@partners.org.
1. Matkovich SJ, Hu Y, Dorn GW. Regulation of cardiac microRNAs by cardiac microRNAs. Circ Res. 2013;113(1):62-71.

2. Song SJ, et al. MicroRNA-antagonism regulates breast cancer stemness and metastasis via TETfamily-dependent chromatin remodeling. Cell. 2013;154(2):311-324.

3. Shi $\mathrm{H}$, et al. Walking the interactome to identify human miRNA-disease associations through the functional link between miRNA targets and dis- ease genes. BMC Syst Biol. 2013;7:101.

4. Lai X, Bhattacharya A, Schmitz U, Kunz M, Vera J, Wolkenhauer O. A systems' biology approach to study microRNA-mediated gene regulatory networks. Biomed Res Int. 2013;2013:703849.

5. Gurtan AM, Sharp PA. The role of miRNAs in regulating gene expression networks. J Mol Biol. 2013;425(19):3582-3600.

6. White K, Loscalzo J, Chan SY. Holding our breath: The emerging and anticipated roles of
microRNA in pulmonary hypertension. Pulm Circ. 2012;2(3):278-290.

7. Courboulin A, et al. Role for miR-204 in human pulmonary arterial hypertension. J Exp Med. 2011;208(3):535-548.

8. Kim J, et al. An endothelial apelin-FGF link mediated by miR-424 and miR-503 is disrupted in pulmonary arterial hypertension. Nat Med. 2013;19(1):74-82.

9. Parikh VN, et al. MicroRNA-21 integrates patho- 
genic signaling to control pulmonary hypertension: results of a network bioinformatics approach. Circulation. 2012;125(12):1520-1532.

10. Barabasi AL, Gulbahce N, Loscalzo J. Network medicine: a network-based approach to human disease. Nat Rev Genet. 2011;12(1):56-68.

11. Friedman RC, Farh KK, Burge CB, Bartel DP. Most mammalian mRNAs are conserved targets of microRNAs. Genome Res. 2009;19(1):92-105.

12. Newman ME. Modularity and community structure in networks. Proc Natl Acad Sci US A. 2006;103(23):8577-8582.

13. Lu Z, et al. miR-301a as an NF- $\mathrm{KB}$ activator in pancreatic cancer cells. EMBO J. 2011; 30(1):57-67.

14. Kovaleva V, et al. miRNA-130a targets ATG2B and DICER1 to inhibit autophagy and trigger killing of chronic lymphocytic leukemia cells. Cancer Res. 2012;72(7):1763-1772.

15. Hager M, et al. MicroRNA-130a-mediated downregulation of Smad 4 contributes to reduced sensitivity to TGF- $\beta 1$ stimulation in granulocytic precursors. Blood. 2011;118(25):6649-6659.

16. Chen Y, Gorski DH. Regulation of angiogenesis through a microRNA (miR-130a) that downregulates antiangiogenic homeobox genes GAX and HOXA5. Blood. 2008;111(3):1217-1226.

17. Wu WH, et al. MicroRNA-130a mediates proliferation of vascular smooth muscle cells in hypertension. Am J Hypertens. 2011;24(10):1087-1093.

18. Mujahid S, Nielsen HC, Volpe MV. MiR-221 and miR-130a regulate lung airway and vascular development. PLoS One. 2013;8(2):e55911.

19. Hansmann G, et al. An antiproliferative BMP-2/ PPAR $\gamma /$ apoE axis in human and murine SMCs and its role in pulmonary hypertension. J Clin Invest. 2008;118(5):1846-1857.

20. Crossno JT, et al. Rosiglitazone attenuates hypoxia-induced pulmonary arterial remodeling. Am J Physiol Lung Cell Mol Physiol. 2007;292(4):L885-L897.

21. Nisbet RE, et al. Rosiglitazone attenuates chronic hypoxia-induced pulmonary hypertension in a mouse model. Am J Respir Cell Mol Biol. 2010;42(4):482-490.

22. Liu Y, et al. Peroxisome proliferator-activated receptor-gamma ameliorates pulmonary arterial hypertension by inhibiting 5-hydroxytryptamine 2B receptor. Hypertension. 2012;60(6):1471-1478.

23. Rabinovitch M. PPAR $\gamma$ and the pathobiology of pulmonary arterial hypertension. Adv Exp Med Biol. 2010;661:447-458.

24. Alastalo TP, et al. Disruption of PPAR $\gamma / \beta$ - catenin-mediated regulation of apelin impairs BMP-induced mouse and human pulmonary arterial EC survival. J Clin Invest. 2011;121(9):3735-3746.

25. Soubrier F, et al. Genetics and genomics of pulmonary arterial hypertension. J Am Coll Cardiol. 2013;62(25 suppl):D13-D21.

26. Covello KL, et al. HIF-2 $\alpha$ regulates Oct- 4 : effects of hypoxia on stem cell function, embryonic development, and tumor growth. Genes Dev. 2006;20(5):557-570.

27. Firth AL, Yao W, Remillard CV, Ogawa A, Yuan JX. Upregulation of Oct- 4 isoforms in pulmonary artery smooth muscle cells from patients with pulmonary arterial hypertension. Am J Physiol Lung Cell Mol Physiol. 2010;298(4):L548-L557.

28. Ciuclan L, et al. A novel murine model of severe pulmonary arterial hypertension. Am J Respir Crit Care Med. 2011;184(10):1171-1182.

29. Steiner MK, Syrkina OL, Kolliputi N, Mark EJ, Hales CA, Waxman AB. Interleukin-6 overexpression induces pulmonary hypertension. Circ Res. 2009;104(2):236-244.

30. Graham BB, et al. Schistosomiasis-induced experimental pulmonary hypertension: role of interleukin-13 signaling. Am J Pathol. 2010;177(3):1549-1561.

31. West J, et al. Pulmonary hypertension in transgenic mice expressing a dominant-negative BMPRII gene in smooth muscle. Circ Res. 2004;94(8):1109-1114.

32. Reddy V, et al. In utero placement of aortopulmonary shunts. A model of postnatal pulmonary hypertension with increased pulmonary blood flow in lambs. Circulation. 1995;92(3):606-613.

33. Lee EK, et al. miR-130 suppresses adipogenesis by inhibiting peroxisome proliferator-activated receptor gamma expression. Mol Cell Biol. 2011;31(4):626-638

34. Obad S, et al. Silencing of microRNA families by seed-targeting tiny LNAs. Nat Genet. 2011;43(4):371-378.

35. Hansmann G, et al. Pulmonary arterial hypertension is linked to insulin resistance and reversed by peroxisome proliferator-activated receptor-gamma activation. Circulation. 2007;115(10):1275-1284.

36. Hansmann G, Zamanian RT. PPAR $\gamma$ activation: a potential treatment for pulmonary hypertension. Sci Transl Med. 2009;1(12):12ps14.

37. Li, et al. Heme oxygenase-1/p21WAF1 mediates peroxisome proliferator-activated receptor- $\gamma$ signaling inhibition of proliferation of rat pul- monary artery smooth muscle cells. FEBS J. 2010;277(6):1543-1550.

38. Teichert-Kuliszewska K, et al. Bone morphogenetic protein receptor-2 signaling promotes pulmonary arterial endothelial cell survival: implications for loss-of-function mutations in the pathogenesis of pulmonary hypertension. Circ Res. 2006;98(2):209-217.

39. Sakao S, Taraseviciene-Stewart L, Lee J, Wood K, Cool C, Voelkel N. Initial apoptosis is followed by increased proliferation of apoptosis-resistant endothelial cells. FASEB J. 2005;19(9):1178-1180.

40. Wang LH, et al. Transcriptional inactivation of STAT3 by PPAR $\gamma$ suppresses IL-6responsive multiple myeloma cells. Immunity. 2004;20(2):205-218

41. Paulin R, et al. Signal transducers and activators of transcription-3/pim1 axis plays a critical role in the pathogenesis of human pulmonary arterial hypertension. Circulation. 2011;123(11):1205-1215.

42. Malenfant S, et al. Signal transduction in the development of pulmonary arterial hypertension. Pulm Circ. 2013;3(2):278-293.

43. Lee MJ, et al. Sequential application of anticancer drugs enhances cell death by rewiring apoptotic signaling networks. Cell. 2012;149(4):780-794.

44. Chan SY, Loscalzo J. The emerging paradigm of network medicine in the study of human disease. Circ Res. 2012;111(3):359-374.

45. Chan SY, Loscalzo J. Pathogenic mechanisms of pulmonary arterial hypertension. J Mol Cell Cardiol. 2008;44(1):14-30.

46. Hickey MM, et al. The von Hippel-Lindau Chuvash mutation promotes pulmonary hypertension and fibrosis in mice. JClin Invest. 2010;120(3):827-839.

47. Paulin R, Meloche J, Jacob MH, Bisserier M, Courboulin A, Bonnet S. Dehydroepiandrosterone inhibits the Src/STAT3 constitutive activation in pulmonary arterial hypertension. Am J Physiol Heart Circ Physiol. 2011; 301(5):H1798-H1809.

48. Meloche J, et al. Role for DNA damage signaling in pulmonary arterial hypertension. Circulation. 2013;129(7):786-797.

49. Kang BY, Kleinhenz JM, Murphy TC, Hart CM. The PPAR $\gamma$ ligand rosiglitazone attenuates hypoxia-induced endothelin signaling in vitro and in vivo. Am J Physiol Lung Cell Mol Physiol. 2011;301(6):L881-L891.

50. Wei C, et al. Circulating miRNAs as potential marker for pulmonary hypertension. PLoS One. 2013;8(5):e64396. 